ARTICLE

https://doi.org/10.1038/s41467-019-10753-5

\title{
The microRNAs miR-204 and miR-211 maintain joint homeostasis and protect against osteoarthritis progression
}

Jian Huang (1) 1,3, Lan Zhao (1) 1,3, Yunshan Fan ${ }^{1}$, Lifan Liao ${ }^{1}$, Peter X. Ma (1) ${ }^{2}$, Guozhi Xiao ${ }^{1}$ \& Di Chen ${ }^{1}$

Osteoarthritis (OA) is a common, painful disease. Currently $O A$ is incurable, and its etiology largely unknown, partly due to limited understanding of $O A$ as a whole-joint disease. Here we report that two homologous microRNAs, miR-204 and miR-211, maintain joint homeostasis to suppress OA pathogenesis. Specific knockout of miR-204/-211 in mesenchymal progenitor cells (MPCs) results in Runx2 accumulation in multi-type joint cells, causing whole-joint degeneration. Specifically, miR-204/-211 loss-of-function induces matrix-degrading proteases in articular chondrocytes and synoviocytes, stimulating articular cartilage destruction. Moreover, miR-204/-211 ablation enhances NGF expression in a Runx2-dependent manner, and thus hyper-activates Akt signaling and MPC proliferation, underlying multiplex non-cartilaginous OA conditions including synovial hyperplasia, osteophyte outgrowth and subchondral sclerosis. Importantly, miR-204/-211-deficiency-induced OA is largely rescued by Runx2 insufficiency, confirming the miR-204/-211-Runx2 axis. Further, intraarticular administration of miR-204-expressing adeno-associated virus significantly decelerates OA progression. Collectively, miR-204/-211 are essential in maintaining healthy homeostasis of mesenchymal joint cells to counteract OA pathogenesis.

\footnotetext{
${ }^{1}$ Department of Orthopedic Surgery, Rush University Medical Center, Chicago, IL 60612, USA. ${ }^{2}$ Department of Biologic and Materials Science, University of Michigan, Ann Arbor, MI 48109, USA. ${ }^{3}$ These authors contributed equally: Jian Huang, Lan Zhao. Correspondence and requests for materials should be addressed to D.C. (email: di_chen@rush.edu)
} 
O steoarthritis (OA) is a painful joint disease affecting more than $10 \%$ of the adult population ${ }^{1,2}$. Pathological changes of OA embody multiple manifestations such as articular cartilage (AC) destruction, synovial hyperplasia, osteophyte formation, and subchondral bone sclerosis. In addition, OA patients often show varied symptoms and respond differently to clinical interventions, suggesting that $\mathrm{OA}$ is a heterogeneous disease and its management demands therapeutics that offer greater precision 3,4 . Currently available studies ascribe the pathogenic causes of OA to dysregulation of anabolic and catabolic pathways that affect cartilage matrix maintenance and bone remodeling. However, clinical trials targeting related factors such as matrix metalloproteinases (MMPs), inflammatory cytokines, or growth factors, showed mixed results regarding the efficacy or safety of the therapeutics 5,6 . Thus, development of efficacious OA therapies calls for more in-depth understanding of OA and should benefit from identification of new molecular mechanisms that underlie the complex and multifaceted phenotypes of OA.

Multiple joint tissues undergo structural and functional failure during OA pathogenesis, including AC, meniscus, and synovium. These tissues are mesenchymal or contain significant portions of mesenchymal cells. Moreover, mesenchymal stem cells (MSCs) and mesenchymal progenitor cells (MPCs) are thought to exist in these joint tissues and play important roles in joint homeostasis and maintenance ${ }^{7}$. Importantly, the formation of osteophytes, a prominent radiographic feature of OA and a significant source of pain and joint dysfunction, requires MPCs derived from adjacent areas including periosteum and synovium to recapitulate endochondral ossification ${ }^{8}$. As miRNAs emerge as important regulators in skeletal pathophysiology 9,10 , their roles in MPCderived skeletal tissues remain elusive. Through in vitro work we identified two homologous microRNAs, $m i R-204$ and $m i R-211$, which negatively regulate Runx2 in $\mathrm{MPCs}^{11}$. However, the in vivo functions of $m i R-204 /-211$ are largely unknown, especially regarding how they regulate MPCs and the pathophysiology of mesenchyme-derived skeletal tissues like synovial joints.

Here we identify a mechanism by which $m i R-204 /-211$ regulate the proliferation and differentiation of MPCs in the entire joint and maintain healthy metabolism of joint tissues, thus helping the joint to counteract OA pathogenesis in a well-rounded manner. Our study takes advantage of in vivo analysis of genetically modified mice with $m i R-204 /-211$ deficiency in MPCs and their descendent cells and we reveal that miR-204/-211-Runx2 axis is pivotal in maintaining joint tissue homeostasis. Dysregulation of this signaling pathway leads to multifactorial pathogenesis of $\mathrm{OA}$, particularly with respect to noncartilaginous pathological anabolic changes such as synovial hyperplasia and osteophyte formation. In addition, our results demonstrate that intraarticular reintroduction of $m i R-204$ alleviates OA progression in a surgically induced OA model.

\section{Results}

Global deficiency of $m i R-204 /-211$ induces OA. Our in vitro data showed that two homologous miRNAs, miR-204 and miR211, suppress Runx2 expression in MPCs ${ }^{11}$. However, little is known about their in vivo functions. Thus, we have generated mouse models with conditional knockout (cKO) alleles for $m i R$ 204 and $m i R-211$. A diagram of the targeting vector for $m i R-204$ or $m i R-211$ is shown in Fig. 1a. To remove the lacZ and Neo cassettes, we crossed the targeted mice with flippase-expressing transgenic mice, and thus generated floxed alleles for the miRNAs (Fig. 1a, Supplementary Fig. 1a). Since $m i R-204$ or $m i R-211$ was reported to be expressed in a variety of tissues and play diverse pathophysiological roles ${ }^{12-16}$, we were interested to investigate if genetically modified animals with global deletions of both
miRNAs, which were not yet available but are essential to elucidation of the miR-204/-211 functions, showed any aberrant phenotypes. Thus, we crossed CMV-Cre transgenic mice ${ }^{17}$ with $m i R-204^{\text {flox/flox }}$ and $m i R-211^{\text {flox/flox }}$ mice to generate global knockout (KO) of both miRNAs (Supplementary Fig. 1b, c), namely the mice genotyped as $m i R-204^{-1-}$; miR-211 $1-$ mice. These $m i R-204 /-211$-deficient mice did not show very apparent abnormality postnatally and postpubertally. However, our preliminary analysis of older global $m i R-204 /-211 \mathrm{KO}$ mice through the Mouse Metabolic Phenotyping Center at University of California, Davis has revealed a spectrum of pathological changes such as hypermature cataracts with rupture, uveal melanosis and melanosis, retinal dysplasia, follicular dysplasia, valvular endocardiosis, etc. (Supplementary Table 1).

We also looked at the knee joint phenotype of the global $\mathrm{KO}$ mice. At the age of 9 weeks, the $m i R-204^{-1-}$; miR-211 ${ }^{-1-}$ mice did not differ from the wild type in joint histology, particularly in terms of the tibial growth plate length and AC thickness (Supplementary Fig. 1d, f, g). At the age of 15 weeks, the miR$204^{-l-} ; \mathrm{miR}-211^{-l-}$ mice still did not show difference in growth plate length, but started to possess increased AC abrasion (Supplementary Fig. 1e, h). Strikingly, examination of the aged

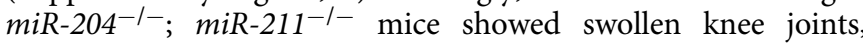
which was demonstrated by $\mu \mathrm{CT}$ analysis to contain ectopically grown bone spurs, resembling osteophyte outgrowth in human OA patients (Fig. 1b). The structural deterioration in the knee joints of the mutant mice involved multiple types of tissues inside the joint, as displayed by highly abrasive AC, subchondral bone thickening, severe synovial hyperplasia, excessive osteophyte formation, and meniscus calcification (Fig. 1c-f). As the pathologic condition of human OA disease and a wellestablished OA model ${ }^{18}$ (Supplementary Fig. 1i) embodies all of these histological changes, we concluded that the miR-204/-211deficient mice had undergone OA onset and progression.

Deletion of $m i R-204 /-211$ in MPCs promotes OA pathogenesis. The tissues affected by OA pathogenesis in the $m i R-204 /-211 \mathrm{KO}$ mice include AC, meniscus, subchondral bone, synovium and joint capsules, of which all are mesenchymal tissues. This suggested that the phenotype observed in the $m i R-204 /-211 \mathrm{KO}$ mice could be caused by the loss of $m i R-204 /-211$ in the mesenchymal stem/progenitor cells, which give rise to these joint tissues. Thus, we decided to specifically delete $m i R-204 /-211$ in MPCs by crossing the miR-204flox/flox and miR-211 flox/flox mice with Prx1Cre transgenic mice, in which the Prx 1 promoter was reported to drive Cre expression in the early limb bud mesenchyme and in the MPCs ${ }^{19,20}$.

Single KO mice for miR-204 (miR-204flox/flox; Prx1-Cre) or miR-211 (miR-211 flox/flox; Prx1-Cre) did not show obvious joint phenotype (Supplementary Fig. 2a, b). Thus, we focused on breeding and analyzing double $\mathrm{KO}$ mice $\left(m i R-204^{\text {flox/flox; }}\right.$ miR211flox/flox; Prx1-Cre, hereafter dKO). Interestingly, we had observed a significantly lower birth ratio of the $\mathrm{dKO}$ mice than that of the littermates without Prx1-Cre. This prompted us to perform a whole-genome sequencing, which revealed that the Prx1-Cre transgene is located on Chromosome 7 (Supplementary Fig. 2c) where $m i R-211$ also resides. Thus, the lower ratio of the $\mathrm{dKO}$ mice was largely contributed by the genetic linkage between miR-211 and transgenic Prx1-Cre. Meanwhile, we have not observed significant gross abnormality of the dKO pups, which survived as well as their Prx1-Cre- littermates, suggesting that the ablation of $m i R-204 /-211$ has no apparent impacts on skeletal development and growth in the young mice.

We examined the $\mathrm{dKO}$ mice to identify if the OA phenotype caused by a global deficiency of $m i R-204 /-211$ could be 
a
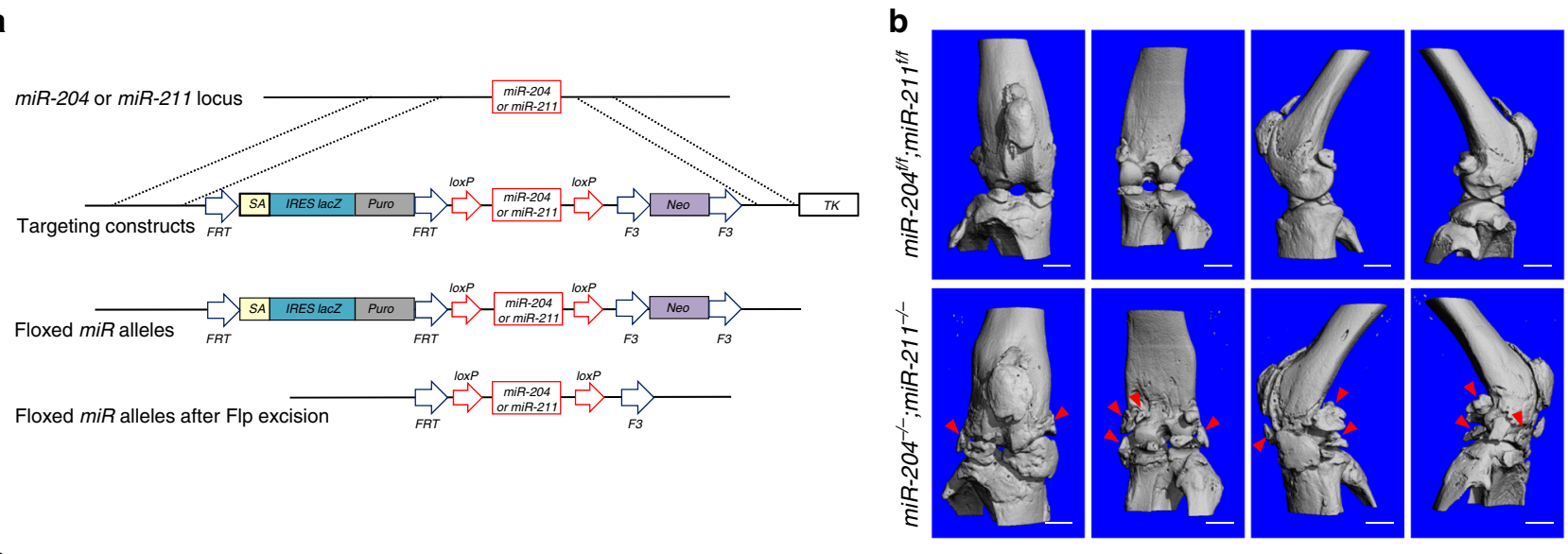

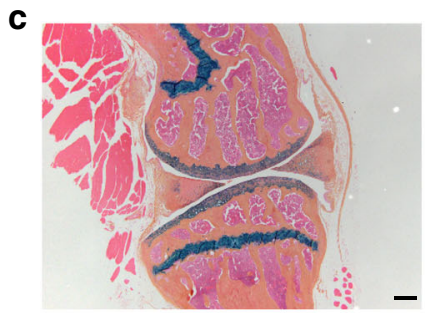

$\operatorname{miR}-204^{\mathrm{f} / \mathrm{f}} ; \operatorname{miR}-211^{\mathrm{f} / \mathrm{f}}$

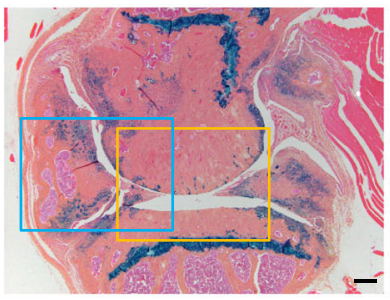

miR-204 ${ }^{-/} ; \operatorname{miR}-211^{-/-}$

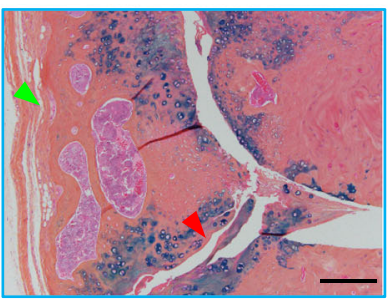

$\operatorname{miR}-204^{-/} ; \operatorname{miR}-211^{-/-}$

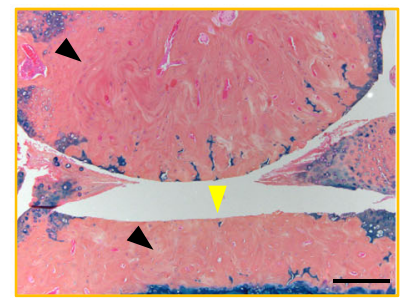

miR-204 $4^{-/} ; m i R-211^{-/-}$ d

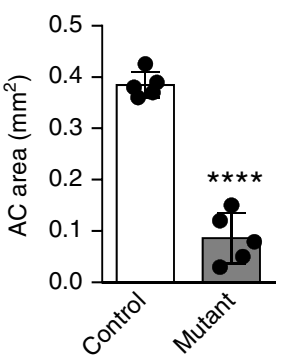

e

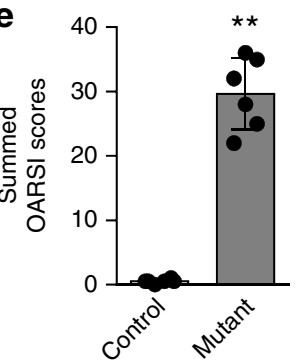

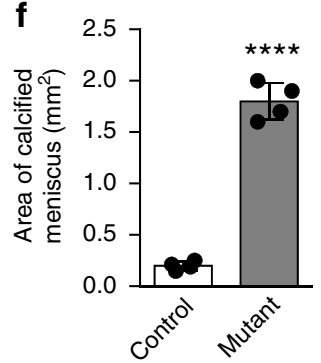

Fig. 1 Germline deletions of miR-204/-211 cause multifaceted OA phenotype. a Schematic of miR-204 (or miR-211) conditional knockout (cKO) allele. Note that the contents, such as lines, boxes and arrows, are not proportional. b Representative $\mu \mathrm{CT}$ images of 46-week-old miR-204/-211 floxed (control) and global double knockout mice (miR-204-/-; miR-211-/-). Red arrowheads, osteophytes. $n=5$. Scale bar, 1 mm. c Representative histology images of knee joints of 46-week-old miR-204/-211 floxed mice and global double KO (miR-204-/-; miR-211-/-) mice stained by Alcian blue/Orange G. Yellow arrowhead, loss of articular cartilage; red arrowhead, osteophytes; green arrowhead, synovial hyperplasia; black arrowheads, subchondral sclerosis. $n=5$. Scale bar, $200 \mu$ m. d, $\mathbf{f}$ Histomorphometric quantification of medial AC area (d), and calcified meniscus area (f) of 46-week-old miR-204/-211 floxed mice (control) and miR-204-I-; miR-211-/- mice (mutant). ${ }^{\star \star \star \star} P<0.0001, n=4$ or 5 . Unpaired Student's $t$ test. e Osteoarthritis research society international (OARSI) scoring system was used to evaluate knee joint articular cartilage (AC) destruction in 46-week-old control and mutant mice. ${ }^{\star \star} P<0.01$, Mann-Whitney test. $n=6$

recapitulated by the loss of the miRNAs in MPCs. The $\mu \mathrm{CT}$ scanning of the knee joints of 42 -week-old mice revealed striking osteophyte growth (Fig. 2a). Further histology analysis of the $\mathrm{dKO}$ mice confirmed strong OA-like phenotypes, including synovial hyperplasia, cartilage degradation, subchondral sclerosis, osteophyte formation, and narrowed knee joint space (Fig. 2b). Particularly, the OA phenotypes developed gradually with age, as exemplified by 42 -week-old mice showing more severe synovial hyperplasia and cartilage degradation than 33-week-old mice (Fig. 2b). It is notable that osteophytes were accompanied by more ectopic cartilage formation (blue staining) in 33-week-old mice than in 42-week-old mice, suggesting that osteophyte growth was preluded by excess cartilage formation (Fig. 2b). In other words, osteophyte growth in the $\mathrm{dKO}$ knee joints resulted from de novo endochondral ossification that requires cartilaginous templates derived from MPCs. Osteoarthritis Research Society International (OARSI) scoring ${ }^{21}$ also demonstrated more severe cartilage destruction in the $\mathrm{dKO}$ mice and a progressive
OA development (Supplementary Fig. 2d). As pain is the most common individual burden for $\mathrm{OA}$ patients and the most prominent symptom of OA, we also performed behavioral pain tests and found that the dKO mice had increased pain sensitivity compared with Prx1-Cre-negative littermates (Fig. 2c). Thus, our results suggested that the deficiency of $m i R-204 /-211$ in MPCs and mesenchymal joint tissues contributed to the genesis of OA pain, possibly through the structural deterioration of the joint.

Next, we performed in situ hybridization (ISH) assays with highly specific locked nucleic acid (LNA)-enhanced miRNA probes to determine the expression of $m i R-204$ and $m i R-211$ in the control and dKO mice. Our results demonstrated that both $m i R-204$ and $m i R-211$ are expressed in knee joint tissues, such as $\mathrm{AC}$, meniscus and synovium, which all have mesenchymal origin (Fig. 2d, e, Supplementary Fig. 2e, f). As well, immunohistochemistry (IHC) assays showed that the $\operatorname{Prx} 1$ promoter drives Cre expression in AC, meniscus and synovium, suggesting a comprehensive deletion of $m i R-204 /-211$ in joint tissues by 
a

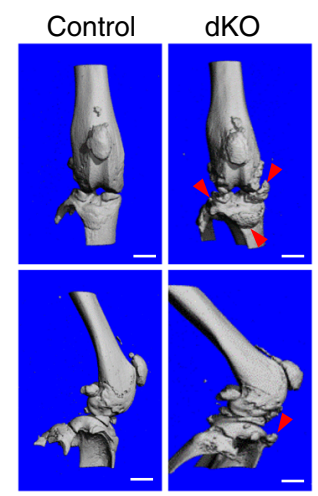

b

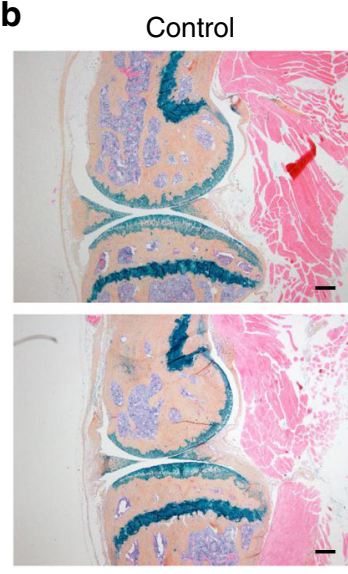

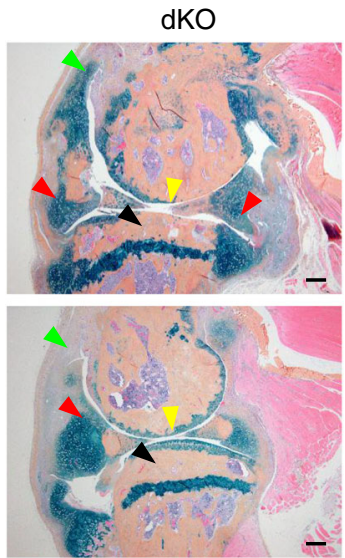

$42 \mathrm{~W}$

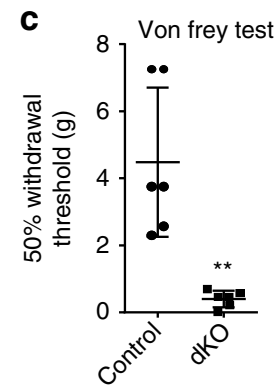

d
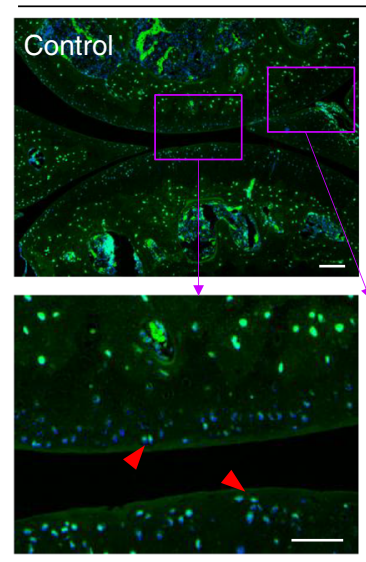

miR-204 in situ hybridization
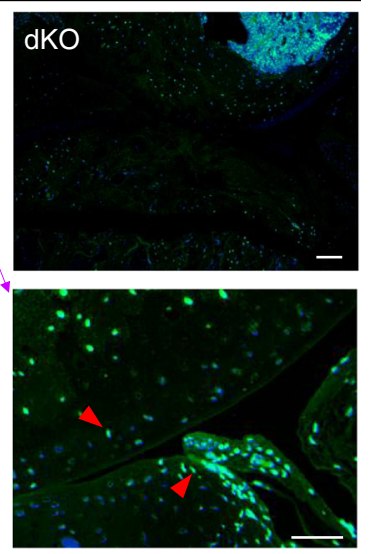

e
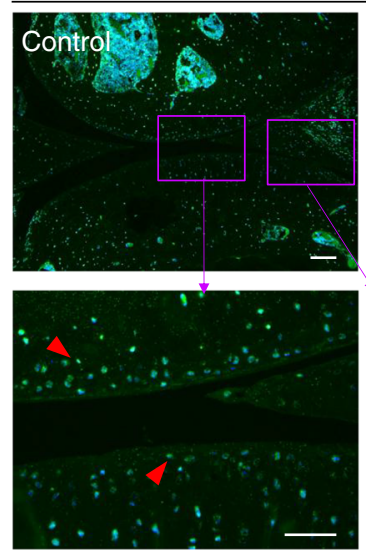

$33 \mathrm{~W}$

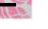
$-1$

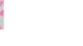

\section{$f$}
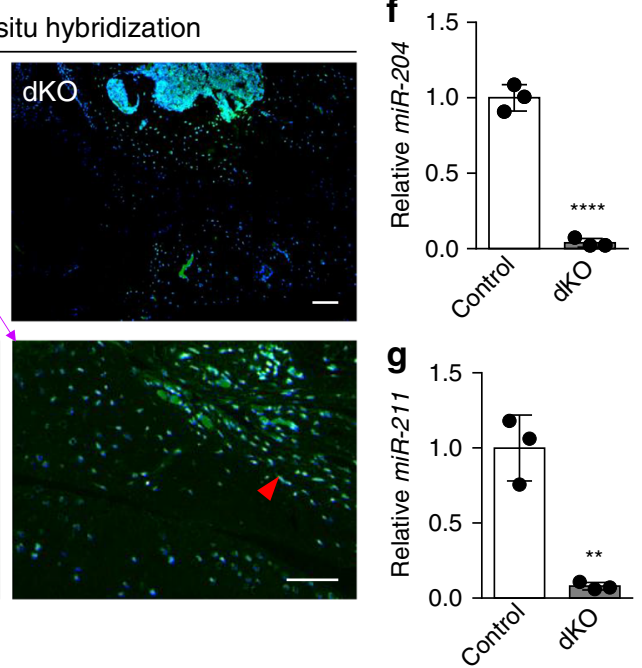

Fig. 2 Deficiency of miR-204/-211 in mesenchymal progenitor cells recapitulates OA pathogenesis. a Representative $\mu$ CT images of 42-week-old miR-204/211 floxed (control) and conditional double KO (dKO, with Prx1-Cre) mice. Red arrowheads, osteophytes. $n=5$. Scale bar, $1 \mathrm{~mm}$. b Representative histology images of knee joints in 42- (top) or 33-week-old (bottom) control and dKO mice stained by Alcian blue/Orange G. Yellow arrowheads, loss of articular cartilage; red arrowheads, osteochondrophytes; green arrowheads, synovial hyperplasia; black arrowheads, subchondral sclerosis. $n=5$. Scale bar, $200 \mu$ m. c Results of von Frey test on 33-week-old control and dKO mice. $n=6 .{ }^{\star \star} P<0.01$, unpaired Student's $t$ test. d, e In situ hybridization using highly specific LNA-enhanced miR-204 (d) or miR-211 (e) probe (green) on knee joint sections of 7-month-old mice. The bottom panels are the enlargements of the upper images as indicated. DAPI staining marks nuclei (blue). Red arrowheads, miR-204- or miR-211-positive cells. Scale bar, $100 \mu \mathrm{m}$. $\mathbf{f}, \mathbf{g}$ qRT-PCR analysis of miR-204 and miR-211 expression in CD45- BMSCs from control or dKO mice. ${ }^{\star \star} P<0.01,{ }^{\star \star \star \star} P<0.0001$, unpaired Student's $t$ test. $n=3$. Data are shown as the mean \pm s.d.

Prx1-Cre (Supplementary Fig. 2g). Significantly diminished ISH signals of miR-204 (or miR-211) were displayed in AC, synovium and meniscus of dKO mice (Fig. $2 \mathrm{~d}, \mathrm{e}$ ), suggesting that miR-204 and $m i R-211$ had been effectively and specifically deleted in multiple types of mesenchymal tissues in the joint. Moreover, quantitative RT-PCR (qRT-PCR) assays demonstrated that both $m i R-204$ and $m i R-211$ significantly decreased in RNAs extracted from AC or synovium (Supplementary Fig. $2 \mathrm{~h}$, i), while the gene expression of the miRNA host genes (Trpm3 and Trpm1) had no significant change (Supplementary Fig. 2j, k). To further confirm the ablation of $m i R-204 /-211$ in other mesenchymal tissues particularly bone marrow MPCs, we also isolated and cultured $\mathrm{CD} 45^{-}$bone marrow stromal cells (BMSCs), of which the major fraction was shown to be MPCs ${ }^{22}$ (Supplementary Fig. 3). Subsequent qRT-PCR experiments showed a $90 \%$ reduction of miR-204/-211 in CD45- BMSCs isolated from the dKO mice (Fig. 2f, g). Together, our results confirmed that $m i R-204 /-211$ are effectively deleted in mesenchymal tissues, including AC, meniscus and synovium of the knee joints.
Deficiency of miR-204/-211 elevates Runx2 and OA markers. The CD45- BMSCs deficient of miR-204/-211 may be used for the confirmation of the interacting machinery between $m i R-204 /$ 211 and Runx2 mRNA at the molecular level. Thus, we applied the cross-linking and immunoprecipitation (CLIP) technique ${ }^{23}$ to purify the complex comprising Argonaute (AGO) proteins, Agobound miRNAs and miRNA-targeting mRNAs ${ }^{24}$. Our results demonstrated that $m i R-204 /-211$ bind to Runx2 mRNA at two binding sites (Supplementary Fig. 4a). Furthermore, ablation of $m i R-204 /-211$ in $\mathrm{CD}^{-} 5^{-}$BMSCs significantly decreased the ligated chimera between miR-204/-211 and Runx2 mRNA (Supplementary Fig. 4b), suggesting that miR-204/-211 deletion impaired the formation of the AGO-miR-204/-211-Runx2 mRNA tertiary complex and therefore relieved Runx 2 suppression by miR-204/-211.

In addition, our IHC results showed that protein levels of Runx2 were significantly elevated in AC, meniscus and synovium, especially in thickened synovium and osteophyte tissues (Fig. 3a, Supplementary Fig. 4c). Besides mesenchymal tissues in knee 
a
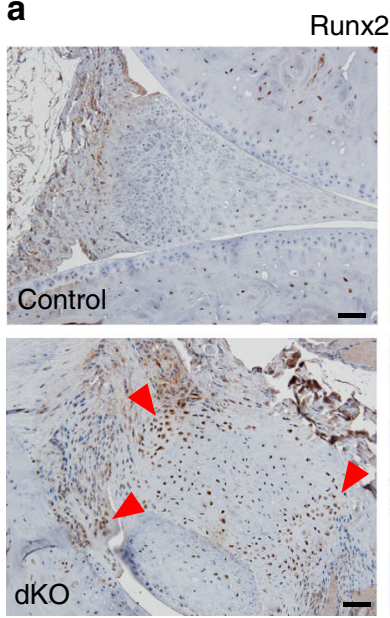

b

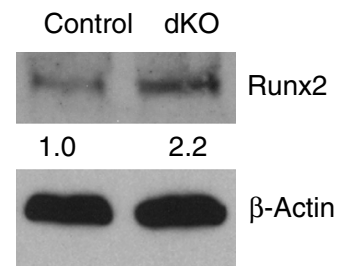

d

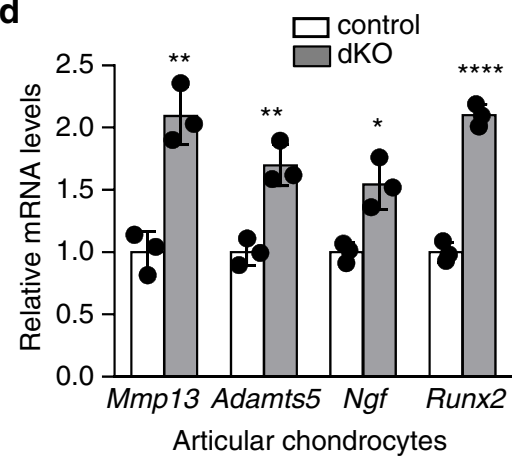

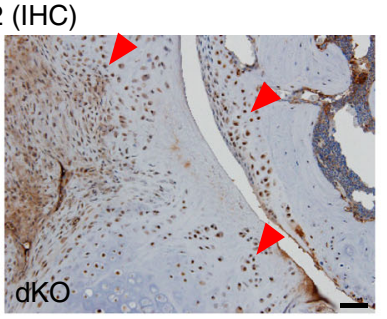

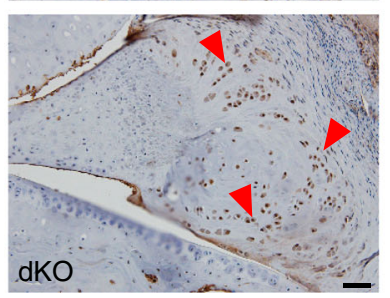

e

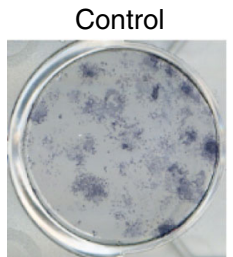

f

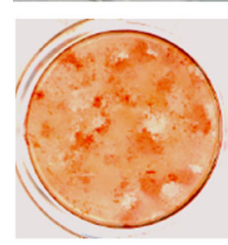

g

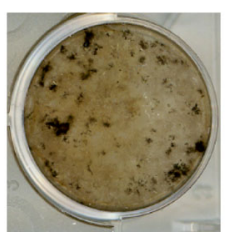

C
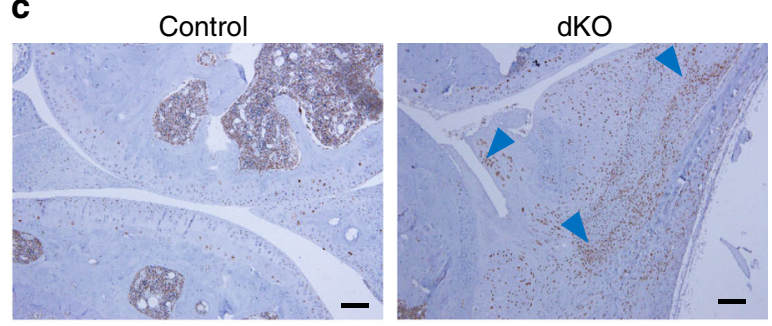

MMP13
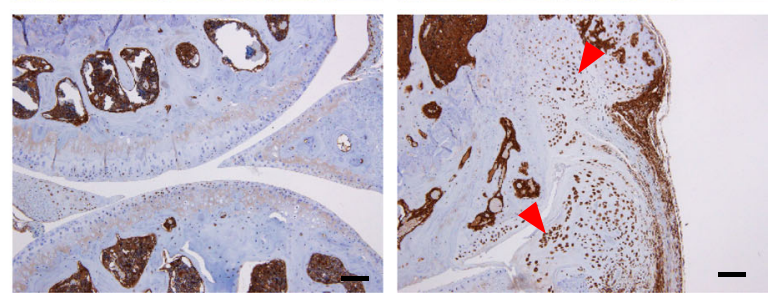

Adamts5

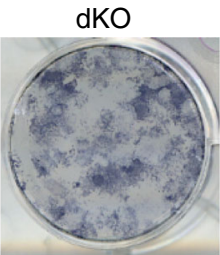

$\mathbf{h}$
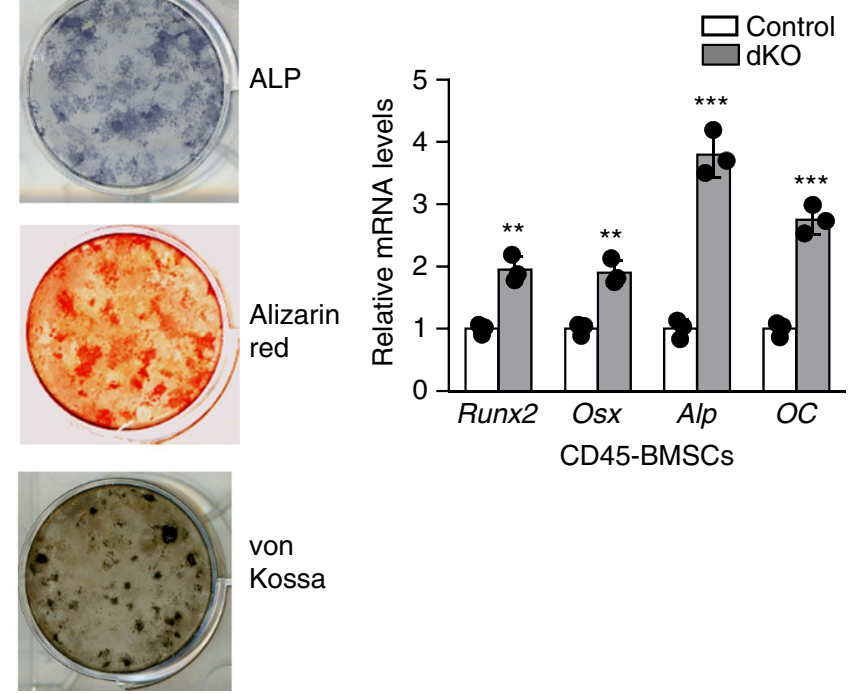

von

Kossa

Fig. 3 Deficiency of miR-204/-211 upregulates Runx2, cartilage-catabolic enzymes and osteogenic markers. a IHC results show enhanced Runx2 expression in the synovium, articular cartilage (AC), and meniscus of knee joints of dKO mice. Red arrowheads, Runx2-positive cells. Scale bar, $50 \mu \mathrm{m}$. $\mathbf{b}$ Western blot analysis shows Runx2 accumulation in CD45- BMSCs isolated from dKO mice. The numbers below the blot indicate the relative protein levels of Runx2 (normalized to $\beta$-actin) in each group compared to the control. $n=3$. c IHC results of MMP13 and Adamts5 in control and dKO mice. Arrowheads, IHCpositive cells. Scale bar, $100 \mu \mathrm{m}$. d qRT-PCR analysis of Mmp13, Adamts5, nerve growth factor (NGF), and Runx2 expression in articular chondrocytes from control or dKO mice. Unpaired Student's $t$ test. $n=3$. Data are shown as the mean \pm s.d. $\mathbf{e}-\mathbf{g}$ Alkaline phosphatase (ALP) staining (e), Alizarin red staining (f), and von Kossa staining (g) of BMSCs isolated from control or dKO mice. $n=3$. h qRT-PCR analysis of Runx2, Osterix (Osx), Alp and Osteocalcin (OC) expression in CD45- BMSCs from control or dKO mice. Unpaired Student's $t$ test. $n=3$. Data are shown as the mean $\pm s . d .{ }^{\star} P<0.05,{ }^{\star \star} P<0.01,{ }^{\star \star \star} P<$ $0.001,{ }^{\star \star \star \star} P<0.0001$. Uncropped western blot scans are shown in Supplementary Data 1

joints, $\mathrm{CD}^{-} 5^{-}$BMSCs of the $\mathrm{dKO}$ mice also showed higher expression of Runx2 proteins (Fig. 3b). These results collectively suggest that miR-204/-211 deletion in MPCs caused significant accumulation of Runx2 in a variety of MPC-derived mesenchymal tissues. Runx 2 has been reported to induce matrix-degrading enzymes such as MMP13, Adamts5 and Adamts425-28, which are upregulated during OA development and therefore deemed as OA marker genes. Thus, we performed IHC to determine if the OA phenotype in the $\mathrm{dKO}$ mice is associated with upregulation of cartilage-degrading enzymes. Our results showed that protein levels of MMP13, Adamts5 (Fig. 3c, Supplementary Fig. 4d, e) and Adamts4 (Supplementary Fig. 4f, g) were significantly elevated in joint tissues, such as $\mathrm{AC}$, meniscus and synovium, of the $\mathrm{dKO}$ mice. In addition, we extracted total RNA from AC of knee joint and found that mRNA levels of Mmp13 and Adamts5 increased in the dKO mice, suggesting that Mmp13 and Adamts5 were upregulated at transcriptional level (Fig. 3d), possibly due to elevated Runx2 levels. Together, our results suggest that the ablation of miR-204 and miR-211 in mesenchymal tissues causes Runx2 accumulation and subsequent induction of proteases, including MMP13, Adamts5 and Adamts4, which mediate catabolic activities in AC degradation and contribute to $\mathrm{OA}$ pathogenesis.

$\mathrm{OA}$ is a complex degenerative joint disorder involving not only cartilage catabolism but also anabolic effects including osteophyte formation and subchondral bone sclerosis, two remarkable 
a

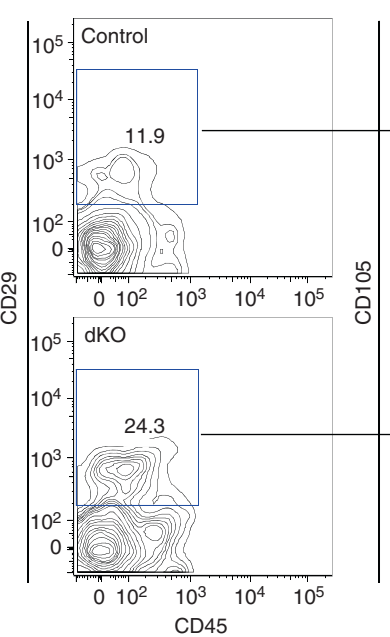

d

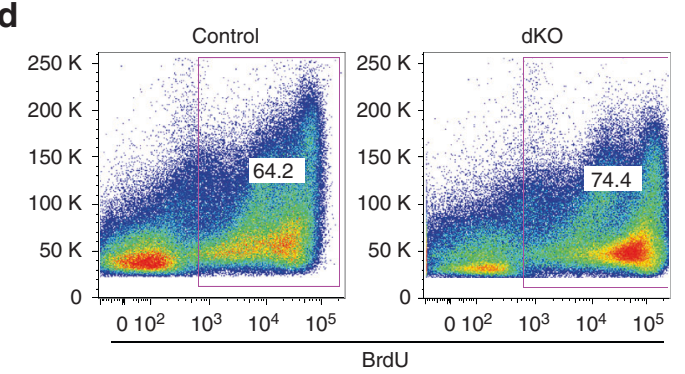

b

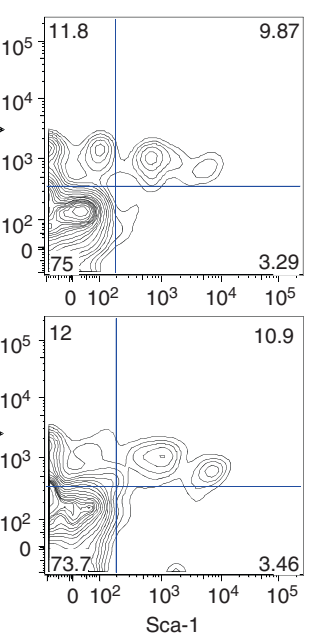

e

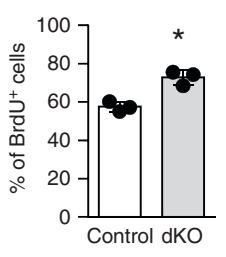

C
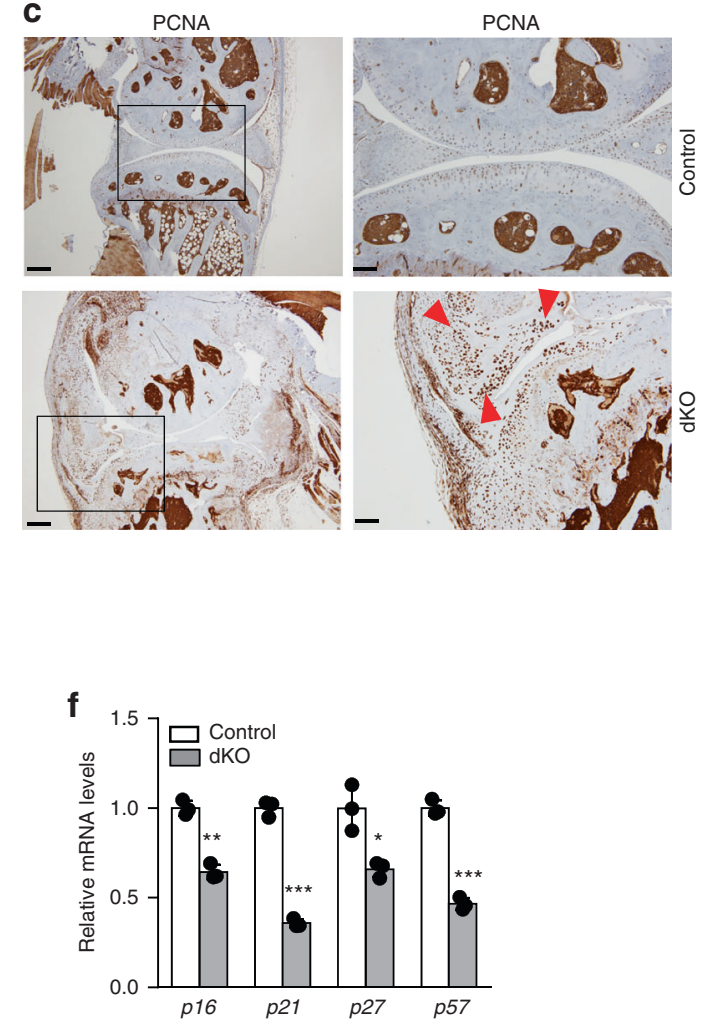

Fig. 4 Proliferation of mesenchymal stem/progenitor cells is enhanced in miR-204/-211 dKO mice. a, b Representative flow cytometry profile (a) and

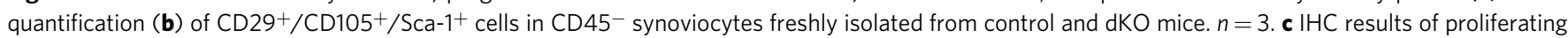
cell nuclear antigen (PCNA) in control and dKO mice. Red arrowheads, PCNA-positive cells. Scale bars (left panels), 250 4 m. Scale bars (right panels), 100 $\mu \mathrm{m}$. d, e Representative flow cytometry profile (d) and quantification (e) of BrdU-labeled CD45- cells, which were in vivo labeled and freshly isolated. $n=$ 3. f Quantitative RT-PCR analysis of p16, p21, p27 and p57 expression in CD45- BMSCs from control or dKO mice. $n=3$. Unpaired Student's $t$ test. Data are shown as the mean \pm s.d. ${ }^{\star} P<0.05,{ }^{\star \star} P<0.01,{ }^{\star \star \star} P<0.001$

exhibitions of aberrantly enhanced osteogenesis. As Runx2 is a master regulator in bone formation, we asked if skeletal stem/ progenitor cells from the $\mathrm{dKO}$ mice, which showed higher Runx2 levels than the cells from the control mice (Fig. 3b), possessed augmented osteogenic activity. We isolated and cultured BMSCs for alkaline phosphatase (ALP), Alizarin red and von Kossa staining, and found that osteoblast differentiation in the cells with miR-204/-211 deletion accelerated (Fig. $3 \mathrm{e}-\mathrm{g}$ ). We also purified CD45- BMSCs from the $\mathrm{dKO}$ mice and found that the mRNA levels of osteoblast markers such as Osterix, Alp and osteocalcin increased markedly in the dKO cells (Fig. 3h), suggesting that loss-of-function of $m i R-204 /-211$ in skeletal progenitor cells promotes osteoblast differentiation. Combined with the IHC data that demonstrated Runx 2 accumulation in joint tissues, our results suggest that increased subchondral bone formation and osteophyte outgrowth could result from enhanced Runx2 levels and elevated osteogenic activity, which are mediated by $m i R-204 /-$ 211 deletion in mesenchymal tissues.

Induced MPC proliferation/synovial hyperplasia in dKO joints. We also observed synovial hyperplasia in the $\mathrm{dKO}$ mice, which represents an important pathological manifestation of OA. As fibroblast-like synovial cells are of mesenchymal origin and display MSC properties such as self-renewal and multipotentiality ${ }^{29}$, we investigated if the deficiency of $m i R-204 /-211$ in MPCs caused aberrant proliferation of mesenchymal cells in synovium, which could underlie hypertrophy of nonhematopoietic tissues in synovium. Firstly, we isolated synovial cells from the dKO mice for flow cytometry to assess the frequency of MPCs in synovial cells. Four cell surface markers were used: CD45 is a hematopoietic cell marker while CD29, CD105 and Sca-1 are markers for $\mathrm{MSCs}^{22}$. The frequencies of $\mathrm{CD} 29^{+} \mathrm{CD} 105^{+} \mathrm{Sca}-1^{+} \mathrm{MSCs}$ in $\mathrm{CD}^{-} 5^{-}$nonhematopoietic synoviocytes were significantly higher in the $\mathrm{dKO}$ mice than those in their $\mathrm{Cre}^{-}$littermates (Fig. $4 \mathrm{a}, \mathrm{b}$ ), suggesting that miR-204/-211-deficient mesenchymal cells in synovium underwent increased proliferation. To assess cell proliferation in the joint tissues, we performed IHC to examine the level of proliferating cell nuclear antigen (PCNA) or Ki-67, and found that positive staining for PCNA and Ki-67 was significantly elevated in the $\mathrm{dKO}$ joint tissues, particularly in hypertrophied synovium, indicating excessive proliferation of synovial cells (Fig. 4c, Supplementary Fig. 5a, b). Also, we injected BrdU into the $\mathrm{dKO}$ mice and performed flow cytometry to evaluate the percentage of BrdU-positive mesenchymal cells in bone marrow. Our results showed that the frequencies of $\mathrm{BrdU}^{+} \mathrm{CD} 45^{-}$cells, which represent proliferating nonhematopoietic cells, were significantly higher in the $\mathrm{dKO}$ mice than in the control mice (Fig. 4d, e), suggesting that mesenchymal cells with the miR-204/211 deficiency retains an enhanced proliferative ability. As well, we observed that the frequencies of $\mathrm{CD} 45^{-} / \mathrm{CD} 29^{+} / \mathrm{CD} 105^{+} / \mathrm{Sca}$ $1^{+}$cells in bone marrow cells were also higher in the dKO mice (Supplementary Fig. 5c, d). Together, our results suggest that $m i R-204 /-211$ ablation results in a greater proliferation rate of MPCs, which provides excessive mesenchymal cells and therefore contributes significantly to synovial hyperplasia in the $\mathrm{dKO}$ mice. 
a

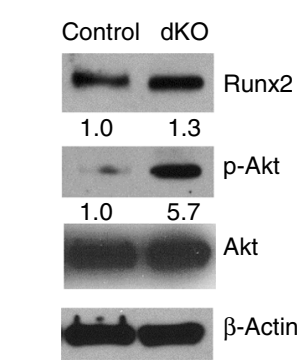

b

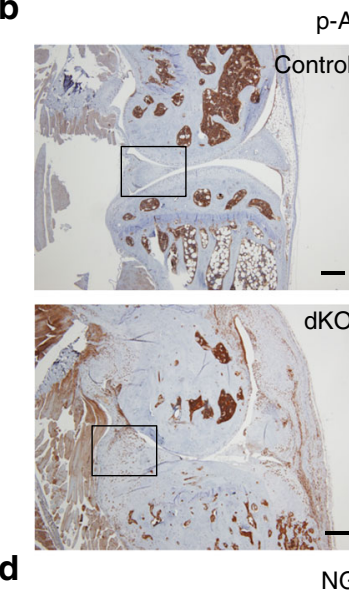

p-AktIHC

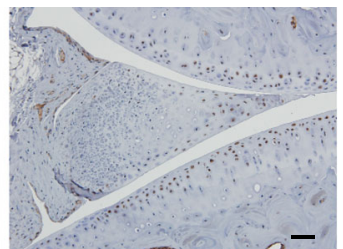

KO

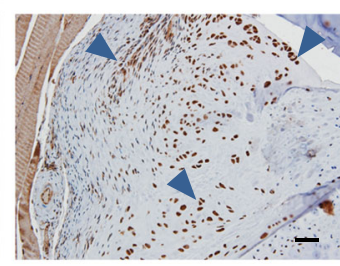

NGF IHC
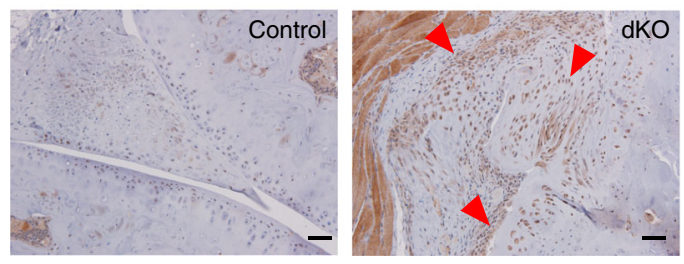

i

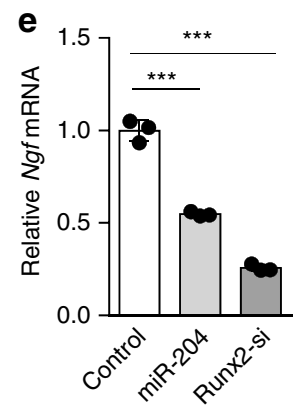

f

GFP NGF-A NGF-B Retrovirus

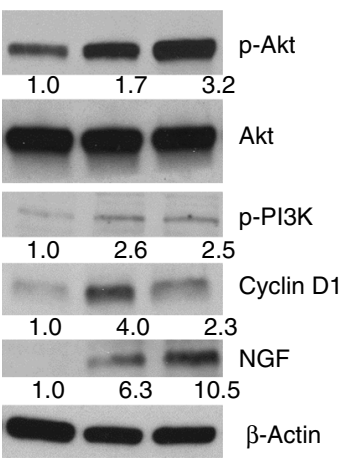

g

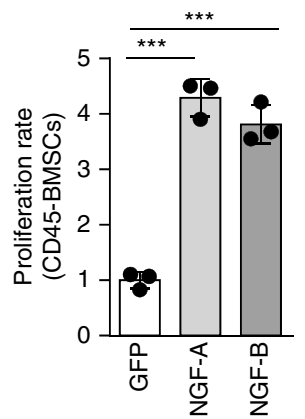

h

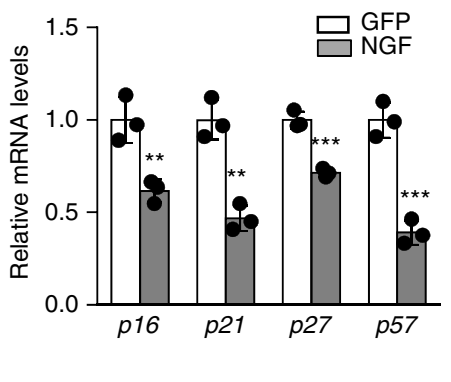

Control Ngf siRNA

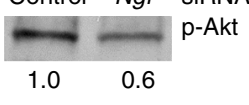

akt

$\beta$-Actin

Fig. 5 The miR-204/-211-Runx2 axis in regulation of NGF-Akt signaling and mesenchymal progenitor cell proliferation. a Western blot analysis shows activated Akt signaling in dKO CD45- BMSCs. The numbers below the blot indicate the relative levels of the denoted protein (except $\mathrm{p}$-Akt normalized to total Akt, all proteins were normalized to $\beta$-actin) in each group compared to the control. $n=3$. $\mathbf{b} \mathrm{IHC}$ results of $\mathrm{p}$-Akt in control and dKO mice. Blue arrowheads mark p-Akt-positive cells. Scale bar, $250 \mu \mathrm{m}$ (left) or $50 \mu \mathrm{m}$ (right). c qRT-PCR analysis of Ngf mRNA expression in CD45- BMSCs from control or dKO mice. ${ }^{\star \star} P<0.01$, unpaired Student's $t$ test. $n=3$. d IHC results of NGF in control and dKO mice. Red arrowheads, NGF-positive cells. Scale bar, $50 \mu \mathrm{m}$. e qRT-PCR analysis of Ngf mRNAs shows that miR-204 or Runx2 siRNA decreases Ngf expression in CD45- BMSCs. ${ }^{\star \star \star P}<0.001$, one-way ANOVA followed by the Tukey-Kramer test. $n=3$. $\mathbf{f}$ Retroviral overexpression of two NGF isoforms in CD45- BMSCs from WT mice activates Akt signaling as shown by western blot. $n=3 . \mathbf{g ~ N G F}$ overexpression increases proliferation of CD45- BMSCs determined by cell number counts. ${ }^{\star \star \star} P<0.001$, one-way ANOVA. $n=3$. h qRT-PCR analysis of CDK inhibitors in mouse CD45- BMSCs with GFP or NGF overexpression. ${ }^{\star \star} P<0.01,{ }^{\star \star \star} P<0.001$, unpaired Student's $t$ test. $n=3$. i Western blot analysis shows that Ngf knockdown downregulates Akt signaling in mouse CD45- BMSCs. $n=3$. All data are shown as the mean \pm s.d. Uncropped western blot scans are shown in Supplementary Data 1

While our results demonstrated indispensable roles of $m i R$ 204/-211 in modulating mesenchymal cell differentiation and catabolism to ameliorate cartilage degradation and ectopic bone formation, our data also revealed an unanticipated facet of $m i R-204 /-211$ regulation that could be related to the proliferation of MPCs. To further investigate the mechanism altering the proliferation of the $\mathrm{dKO}$ cells, we analyzed mRNA expression of cyclin-dependent kinase (CDK) inhibitors, such as $p 16, p 21, p 27$ and $p 57$, in CD45- BMSCs derived from the $\mathrm{dKO}$ mice. Consistent with increased proliferation of mesenchymal cells as shown by BrdU labeling experiments, the mRNA levels of CDK inhibitors decreased substantially (Fig. 4f), suggesting that $m i R-204 /-211$ loss-of-function has negative effects on CDK inhibitors to promote cell cycle progression in MPCs.
Elevated Akt signaling and NGF expression in dKO joints. Because Akt signaling is reported to be pivotal for cell cycle regulation through blocking transcription of CDK inhibitors ${ }^{30}$, we performed western blot assays to determine if activation of Akt signaling is associated with enhanced proliferation of CD45BMSCs from $m i R-204 /-211$-deficient mice. We found that $m i R$ 204/-211 ablation in CD45- BMSCs dramatically increased the Thr308 phosphorylation of Akt proteins, while leaving the amount of total Akt proteins unchanged (Fig. 5a). We also carried out IHC on joint sections and found higher phospho-Akt level in the $\mathrm{dKO}$ mice than their $\mathrm{Cre}^{-}$littermates, especially in hypertrophied synovium and ectopic bone/cartilage-like structures (Fig. 5b, Supplementary Fig. 6a), suggesting that Akt hyperactivation in mesenchymal tissues contributes to synovial hyperplasia and osteophyte outgrowth observed in the dKO mice. 
Activation of Akt signaling cascade can occur with stimulation of receptor tyrosine kinases (RTK) by their ligands ${ }^{31}$. We found that mRNAs coding for nerve growth factor (NGF) were substantially upregulated in $\mathrm{CD}_{4} 5^{-}$BMSCs derived from $\mathrm{dKO}$ mice (Fig. 5c), which have been demonstrated to be comprised of mainly MPCs (Supplementary Fig. 3). The mRNA expression of $N g$ is abundant in CD $45^{-}$BMSCs ( $\triangle \mathrm{Ct} \approx 5$ compared to $\beta$-actin), suggesting that NGF is an endogenous player in mesenchymal cells. We also performed IHC on the dKO knee joint sections and found that NGF protein levels increased significantly in periarticular tissues, especially in synovium and newly formed ectopic cartilage/bone (Fig. 5d, Supplementary Fig. 6b), suggesting that aberrant increase of NGF, a major mediator of OA pain, is also associated with synovial hyperplasia and osteophyte formation in the $\mathrm{dKO}$ mice. To further confirm that NGF expression is induced by $m i R-204 /-211$ loss-of-function and Runx2 accumulation, we introduced synthetic miR-204 mimics or Runx2 siRNAs into CD45- BMSCs and found that both miR-204 and Runx2 siRNAs suppressed Ngf mRNA expression in CD45BMSCs (Fig. 5e), which may be mediated by the decrease of Runx2 levels by miR-204 or by Runx2 siRNAs (Supplementary Fig. 6c). These results indicate that miR-204 and $m i R-211$ modulates NGF expression, Akt activation and cell proliferation through its regulation of Runx2. Retroviral overexpression of two NGF isoforms, i.e. NGF-A and NGF-B, both activated Akt signaling, as the results of western blots showed elevated levels of p-PI3K, p-Akt and cyclin D1 in NGF-transduced CD45progenitor cells (Fig. 5f). As well, NGF overexpression increased $\mathrm{CD}^{2} 5^{-}$cell proliferation (Fig. 5g). Importantly, the overexpression of NGF in $\mathrm{CD}_{4} 5^{-}$BMSCs significantly inhibited the mRNA expression of CDK inhibitors, such as p16, p21, p27 and $p 57$ (Fig. 5h). Accordingly, the knockdown of $\mathrm{Ngf}$ by its siRNA transfection significantly reduced p-Akt protein levels and cell proliferation rate in $\mathrm{CD}_{4} 5^{-}$BMSCs (Fig. 5i, Supplementary Fig. 6d). Collectively, these results suggested that Runx2 accumulation induced by $m i R-204 /-211$ depletion upregulates $\mathrm{Ngf}$ expression to activate Akt signaling and then to promote MPC proliferation, which may constitute a major source of excessive mesenchymal cells contributing to deleterious anabolic events in OA, including synovial hyperplasia, osteophyte outgrowth and subchondral sclerosis.

Administration of $m i R-204$ alleviates surgery-induced OA. The dKO mice displayed severe, comprehensive OA phenotype, including AC degradation, subchondral sclerosis, osteophyte formation and synovial hyperplasia, suggesting that endogenous miR-204/-211 play a key role in maintaining joint tissue homeostasis and preventing OA development. Thus, we ask if OA could be treated by administrating $m i R-204$, as $m i R-204$ and $m i R$ 211 share redundant functions and overexpression of one miRNA rescues the double knockout of two homologous miRNAs ${ }^{32}$. We performed partial meniscectomy to induce the onset of $\mathrm{OA}^{33}$, and then used intraarticular injection of adeno-associated virus (AAV) serotype $5^{34}$ to express $m i R$-204 in OA-inflicted knee joints. Three months after injection, we could still detect AAV expression in a variety of joint tissues including AC, meniscus and synovium (Supplementary Fig. 7), suggesting that AAV5 administered by intraarticular injection can drive potent and long-lasting gene expression in knee joints.

Next, we performed phenotypic analysis to investigate how $m i R-204$ administration affects OA pathogenesis in the surgical OA model. Notably, $\mu \mathrm{CT}$ analysis showed that injection of AAV5-miR-204 diminished osteophyte growth significantly, compared with that of AAV5-control (Fig. 6a). Further, histological examination confirmed a substantial decrease of osteophyte formation and revealed marked mitigation regarding synovial hyperplasia and cartilage degradation in miR-204injected joints (Fig. 6b, c). In pain-related behavioral tests, mice receiving miR-204 injection showed higher pain thresholds than those receiving control AAV5 injection (Fig. 6d), suggesting that miR-204 reintroduction in OA knee joints not only ameliorated histological features, including AC degradation and osteophyte formation, but also reduced pain, a prominent symptom affecting OA patients. We also used IHC to examine the protein levels of Runx2 and several OA markers, and found that miR-204 administration alleviated the upregulation of Runx2, Adamts5 and MMP13, which was induced by the surgery (Fig. $6 \mathrm{e}-\mathrm{h}$ ). Together, our exploration of miR-204 administration in vivo suggest that miR-204 could act as an effective therapeutic agent to treat $\mathrm{OA}$ in a surgically induced $\mathrm{OA}$ mouse model, through inhibition of Runx2 expression in multiple joint tissues.

The Runx2-miR-204/-211 axis controls OA pathogenesis. Accumulating evidence generated in this study points to $m i R$ 204/-211-regulated Runx2 that acts as a central player in OA onset and progression. To alleviate Runx2 accumulation caused by the ablation of $m i R-204 /-211$ in skeletal cells, we introduced Runx2 haploinsufficiency into the $\mathrm{dKO}$ mice by generating the miR-204flox/flox; miR-21 $1_{\text {flox/flox; }}$ Runx $2^{+/ f l o x}$; Prx1-Cre mice, hereafter tKO. Comparison of tKO and $\mathrm{dKO}$ mice revealed that the OA phenotype observed in the $\mathrm{dKO}$ mice has been largely ameliorated in the tKO mice. Specifically, the tKO mice possessed less degradation of $\mathrm{AC}$ in their knee joints than the dKO littermates, as demonstrated by histological analyses. Moreover, we have observed an effective diminish of synovial hyperplasia and osteophyte outgrowth in the tKO than in the $\mathrm{dKO}$ mice (Fig. 7a, Supplementary Fig. 8). These results suggest that Runx2 insufficiency can significantly mitigate the severe OA phenotype caused by $m i R-204 /-211$ ablation in the skeletal cells.

To investigate if reduction of Runx2 protein levels was a causal factor in OA phenotype alleviation, we performed IHC assays to examine Runx2 protein levels in the tKO mice. Our results showed that Runx2 insufficiency markedly attenuated the Runx2 accumulating effects resulting from $m i R-204 /-211$ deficiency, in AC, meniscus and synovium (Fig. 7b). As Runx2 level returned to normal as similar as that in the control mice, the tKO mice also showed a remarkable extenuation in cartilage-catabolic factors including Adamts5 and MMP13 compared to the dKO littermates (Fig. 7c, d). Thus, our results suggest that heterozygous deficiency of Runx 2 rescued the OA phenotype resulting from $m i R-204 /-211$ deletion and readjusted Runx2 proteins to a normal physiologic level, and therefore significantly mitigated the severe OA phenotype observed in the dKO mice. Further, we conclude that $m i R-204 /-211$ control Runx2 expression and the Runx2-miR-204/-211 axis governs OA pathogenesis.

\section{Discussion}

Currently, many mechanistic OA studies focus on cartilaginous tissues $^{35-38}$, since cartilage is a key component of synovial joints and cartilaginous tissues are exposed to mechanical stress that was thought to be a major risk factor for OA. Therefore, cartilage destruction is deemed to be a landmark event during OA onset and progression. In this study, we have generated a new mouse model in which $m i R-204 /-211$ are deleted in multiple joint tissues and a comprehensive OA-like phenotype is observed in the entire joint but not restricted to cartilage only. For example, we found that the $\mathrm{dKO}$ mice develop severe synovial hyperplasia, which may have instrumental effects on other manifestations of OA, such as induced matrix-degrading enzymes on cartilage breakdown and elevated NGF on increased pain sensitivity and MPC 
a

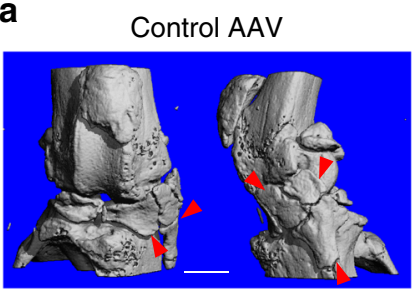

b
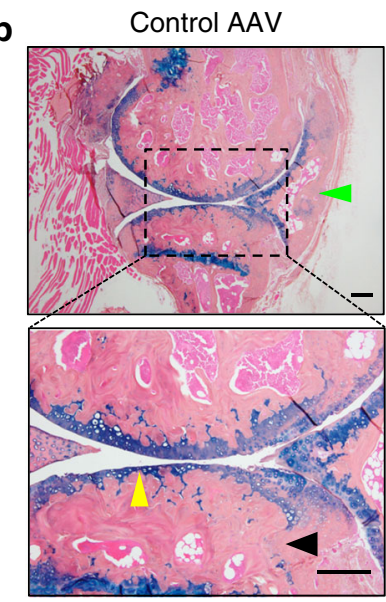

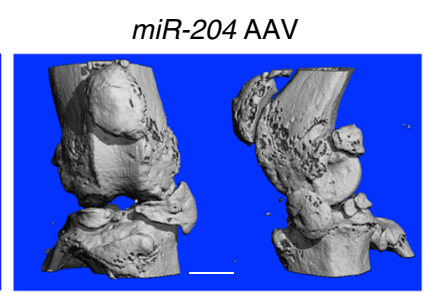

miR-204 AAV
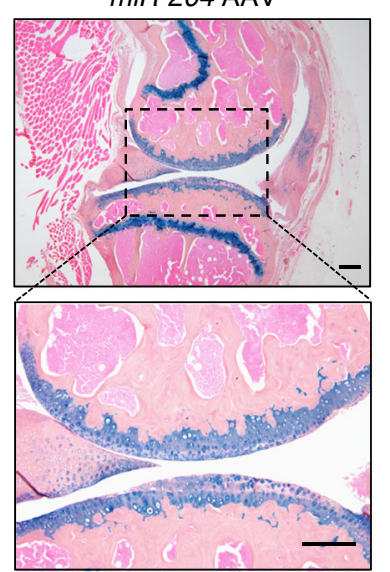

$\mathbf{f}$ e Control AAV
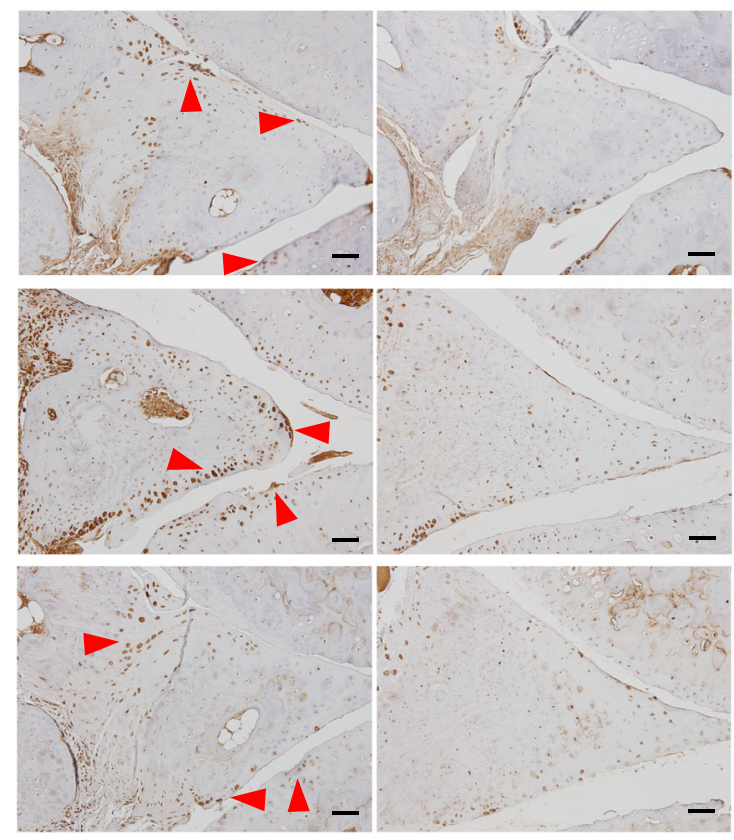

g

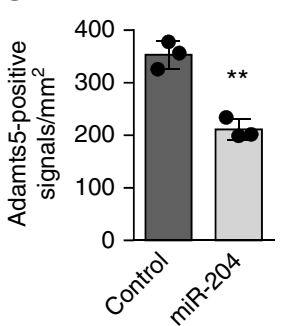

h

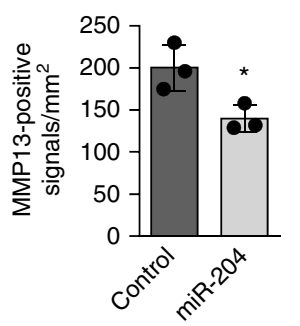

Runx2

Adamts5

MMP13
C

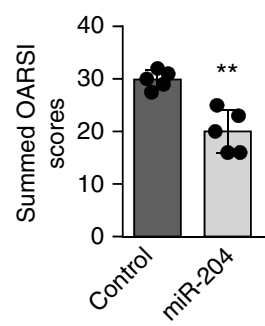

\begin{abstract}
d
\end{abstract}

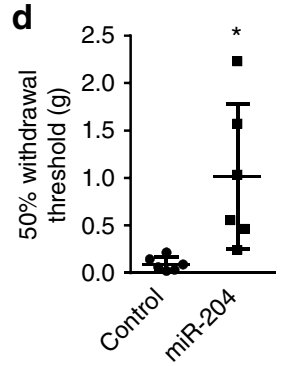

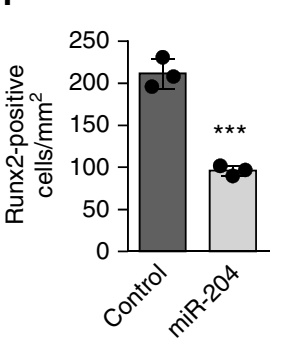

Fig. 6 Intraarticular injections of AAV5-miR-204 alleviates surgically induced OA phenotype. a Representative $\mu$ CT images of osteoarthritic joints in 9-month-old C57BL/ 6 mice receiving AAV5-GFP or AAV5-miR-204 injection at the age of 3 months. Red arrowheads, osteophytes. $n=5$. Scale bar, 1 mm. b Representative histology images of osteoarthritic joints in 9-month-old mice receiving AAV injection at the age of 3 months. Yellow arrowheads, articular cartilage degradation; green arrowhead, synovial hyperplasia; black arrowhead, subchondral sclerosis. $n=5$. Scale bars, $200 \mu$ m. c Analysis using OARSI scoring system was performed to evaluate knee joint AC destruction in mice receiving AAV5-GFP (control) or AAV5-miR-204 injection. $n=5$. Mann -Whitney test. $\mathbf{d}$ Intraarticular injection of AAV5-miR-204 reduces pain sensitivity induced by OA. The von Frey test was performed in 9-month-old mice receiving AAV injection at the age of 3 months. $n=6$. e $\mathrm{IHC}$ results of Runx2, Adamts5, and MMP13 expression in osteoarthritic joints receiving AAV injection. Red arrowheads, IHC-positive cells. Scale bar, $50 \mu \mathrm{m}$. $\mathbf{f}-\mathbf{h}$ Quantification of positive IHC signals per mm ${ }^{2}$ of knee joints for detection of Runx2 (f), Adamtas5 (g), and MMP13 (h). $n=3$. Unpaired Student's $t$ test. ${ }^{\star} P<0.05,{ }^{\star \star} P<0.01,{ }^{\star \star \star} P<0.001$

proliferation. Osteophyte formation is also a prominent feature displayed in the $\mathrm{dKO}$ mice, and we found that osteophyte outgrowth is significantly contributed by excessive proliferation of MPCs, which implicates a mechanistic similarity between osteophyte formation in OA pathogenesis and endochondral ossification in skeletal development. Thus, our dKO mice could represent a comprehensive OA model, of which the analysis provides multifaceted insights into the mechanism of $\mathrm{OA}$ initiation and progression as well as the metabolic maintenance of the joint.

When we were generating the $\mathrm{dKO}$ mice, we had observed a much lower ratio of the dKO pups than that of the miR-204flox/ flox;miR-21 $1^{\text {flox/flox }}$ pups or the miR-204flox/flox; $m i R-211^{+/ f l o x}$; Prxl-Cre pups. This made us wonder if significant prenatal lethality of the dKO mice occurred or the Prx1-Cre transgene is physically linked to the miR-211 gene. Through whole-genome sequencing we have identified that the Prxl-Cre transgene is located on mouse Chromosome 7 (Supplemental Fig. 2c), the same chromosome on which the mouse miR-211 gene also resides. Further we found that the ratio of the dKO pups was largely Mendelian if we crossed a dKO male to a $m i R-204$ flox/flox; miR-21 flox/flox female. These dKO mice did not show apparent abnormalities in young ages and their knee joint phenotypes were not significant at 3-month-old. However, the OA-like features of the dKO mice were aggravated with aging and the severity culminated at the ages of 10-12 months, which is milder than those genetic modified mouse models with disruption of major signaling pathways ${ }^{39-41}$, but bears greater resemblance to human OA. Thus, the use of the Prx1 promoter and the miR-204/-211 deletion at earlier stages in these dKO mice may not greatly affect their development and growth, suggesting that the OA phenotype observed at the later stages ( $>6$ months) may be associated with accrual of miR-deficient effects in aging rather than in embryonic/developmental stages. On the other hand, an extensive deletion of miR-204/-211 in MPCs as well as various types of mesenchymal cells inside the joints demonstrated multifaceted roles of $m i R-204 /-211$ and generated comprehensive pathological 
a

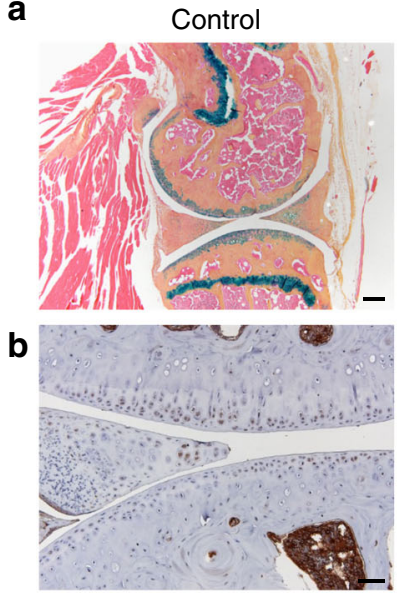

C

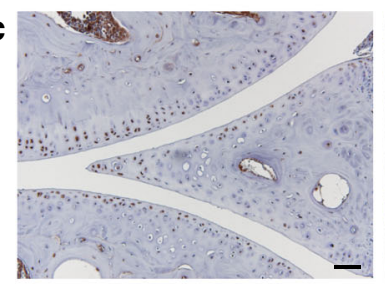

d

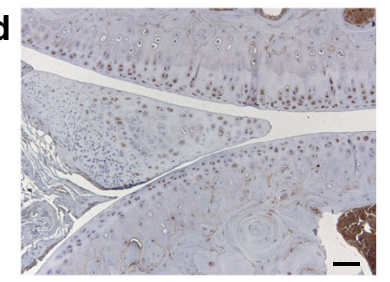

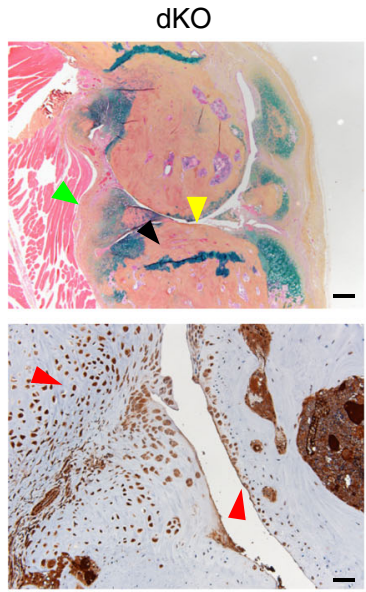
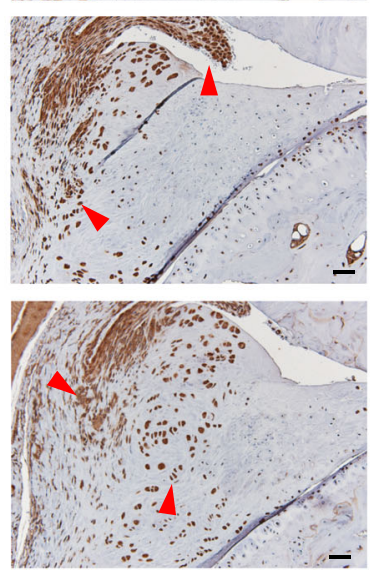

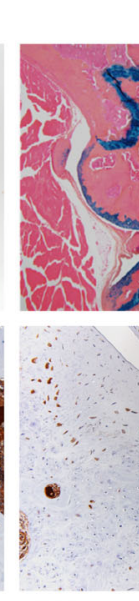

tKO
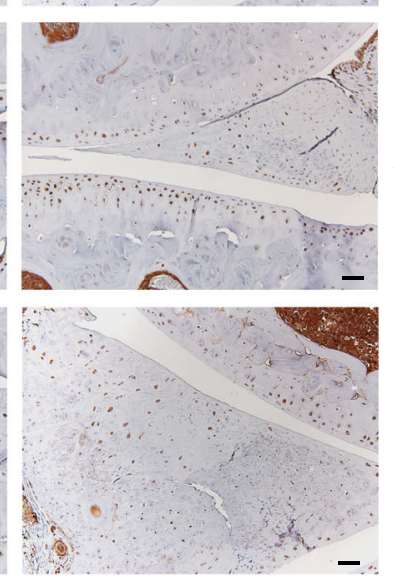

Alcian blue/ orange $\mathrm{G}$

Adamts5

MMP13

Fig. 7 Runx2 insufficiency mitigates OA phenotype in dKO mice. a Representative histology images of 9-month-old tKO (miR-204flox/flox; miR-21fflox/flox; Runx2+/flox; Prx1-Cre) mice compared with dKO (miR-204flox/flox; miR-21flox/flox; Prx1-Cre) and control (miR-204flox/flox; miR-217flox/flox) mice. Yellow arrowheads, articular cartilage degradation; green arrowhead, synovial hyperplasia; black arrowhead, subchondral sclerosis. $n=5$. Scale bars, $200 \mu \mathrm{m}$. b-d IHC results of Runx2 (b), Adamts5 (c), and MMP13 (d) expression in the control, dKO and tKO mice. Red arrowheads, Runx2-, Adamts5-, or MMP13positive cells. Scale bar, $50 \mu \mathrm{m}$

changes not restricted to cartilage degradation. While the use of arguably more cartilage-specific promoters such as those of Aggrecan and Col2a1 would provide cartilage-focused information for OA studies, miRNA ablation in MPCs offers a unique and resourceful perspective which help to advance our understanding of pathological changes of the entire joint.

OA can be regarded as a mesenchymal disease as it affects the entire synovial joint, which contains multiple connective tissues derived from MPCs, or in a broader context, MSCs. In addition, MSCs are reported to be available in most joint tissues, including synovium $^{42}$, meniscus ${ }^{43}$ and cartilage ${ }^{44}$, which act as reservoirs of progenitor cells for tissue maintenance and repair as well as sources of paracrine signaling. Thus, the proper condition of MSCs is vital to the health of diarthrodial joints and OA pathogenesis involves dysregulation of MSC biology. In our study, miR-204/-211 deficiency in MPCs causes detrimental alterations in both proliferation and differentiation, underlying multiple pathological anabolic properties of OA such as synovial hyperplasia, osteophyte outgrowth and subchondral sclerosis. Also, NGF overexpression in MPCs may constitute an important mechanistic cause for inappropriate nerve growth inside joints and resultant OA pain hypersensitivity. Due to the abilities of tissue regeneration and repair of MSCs which help to combat diseases such as $\mathrm{OA}$ and myocardial infarction as well as their immunomodulatory effects to alleviate immune disorders such as multiple sclerosis and Crohn's disease ${ }^{45}$, the numbers of registered clinical trials of MSC-based therapies are rapidly increasing ${ }^{46,47}$. As our study demonstrated that $m i R-204 /-211$ deficiency dysregulates the proliferation and differentiation of MPCs and thus causes OA phenotype, it also highlighted the importance of enhancing the understanding of MSC biology, which will help to develop safer MSC therapy and achieve better clinical outcomes.

In summary, our study of miR-204/-211-deficient mice suggests that the loss-of-function of miR-204/-211 in MPCs leads to aberrant accumulation of Runx2, abnormally increases proliferation and osteogenic differentiation of MPCs, and causes comprehensive OA phenotype. Thus, our results suggest that $m i R-204 /-211$ are essential for the joint to maintain healthy homeostasis and counteract OA pathogenesis.

\section{Methods}

Generation of $\mathbf{m i R - 2 0 4 / - 2 1 1 ~ c K O ~ m i c e . ~ T h e ~ a n i m a l ~ p r o t o c o l ~ o f ~ t h i s ~ s t u d y ~ h a s ~}$ been approved by the Institutional Animal Care and Use Committee (IACUC) of the Rush University Medical Center and all experimental methods and procedures were carried out in accordance with the approved guidelines to comply with all relevant ethical regulations for animal testing and research. To generate $m i R-204 /-$ 211 floxed mice, we engineered the targeting vectors for $m i R-204$ and $m i R-211$ using the recombineering-based method ${ }^{48,49}$. Briefly, an IRES-lacZ reporter cassette as wells as a loxP site were inserted 178 bp or 171 bp upstream miR-204 or $m i R-211$ stem loop and a loxP site plus a Neomycin selection cassette were placed 260 bp or 365 bp downstream miRNA stem loops. Both lacZ and Neo cassettes are flanked by Frt or F3 (a mutant of Frt) sites, allowing the subsequent removal of both cassettes by crossing the chimeric mice to flippase-expressing transgenic mice (The Jackson Laboratory, stock \#: 003946) ${ }^{50}$ and leaving two loxP sites immediately flanking the miRNA stem-loops. Thus, the exogenous sequences brought by the 
targeting of miRNAs are minimal (146 nt at $5^{\prime}$ and $110 \mathrm{nt}$ at $3^{\prime}$ for both floxed alleles) and did not affect the expression or intron splicing of host genes, Trpm3 or Trpm1, of which $m i R-204$ or $m i R-211$ is located in the introns (data not shown). Floxed mice ( $m i R-204^{\text {flox/flox }}$ or $m i R-211^{\text {flox/flox }}$ ) were backcrossed to C57BL/6 for over ten generations. To generate dKO mice ( $m i R-204^{\text {flox/flox; }}$ miR-21 $1_{\text {flox/flox; }}$ Prx 1Cre), male Prx1-Cre transgenic mice ${ }^{19}$ (The Jackson Laboratory, Bar Harbor, ME,

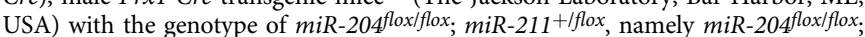
miR-211+lflox; Prx1-Cre, were used to cross to female double floxed mice. To generate germline deletions of the miRNAs, CMV-Cre mice ${ }^{17}$ (The Jackson Laboratory, Bar Harbor, ME, USA) were used.

\section{AAV intraarticular injection and surgically induced OA model. We chose} intraarticular injection of recombinant AAV for in vivo overexpression of $m i R-204$. Both AAV5-EF1a-mmu-mir-204-GFP and AAV5-EF1a-ctrl-GFP were purchased from Vector Biolabs (Malvern, PA, USA). The pri-miR-204 sequence is located in the intron of the EF1a promoter that drives GFP expression, which will be used as a marker for detection of the miR-204 expression introduced by AAV. We performed the surgery of partial meniscectomy on the right knee of wild type (WT) C57BL/6 mice to induce OA 10 days before they turned 3-month-old ${ }^{33}$. Ten days later, we performed intraarticular injection. Specifically, a longitudinal skin incision was made to visualize the patellar ligament and the patella; then we injected $5 \times 10^{9}$ $\mathrm{AAV}$ particles in a $10 \mu \mathrm{l}$ volume into the knee joint cavity by inserting a small needle into the area underneath the patella of the right leg.

Tissue/cell isolation and culture. Mouse synovium were isolated from mouse knee joints ${ }^{51}$. Briefly, a skin incision was made to expose knee joints and quadriceps were resected and reversed distally to remove patella and patellar ligament. As a result, the synovium below patella were exposed for visualization and isolation. For synovial cell isolation, isolated synovium were incubated in $0.1 \% \mathrm{col}$ lagenase D (Roche, Indianapolis, IN, USA) for $15 \mathrm{~min}$. Mouse articular chondrocytes were isolated according to a published protocol ${ }^{52}$ and cultured before analysis. Mouse bone marrow cells were isolated from long bones and cultured for 5 days in a-minimal essential medium ( $\alpha$-MEM) (Life Technologies, Grand Island, NY, USA) with $20 \%$ fetal bovine serum (FBS) (Life Technologies, Grand Island, NY, USA) and then in $\alpha$-MEM with $10 \%$ FBS for a longer duration. CD45- BMSC purification was performed by anti-CD45-mediated negative selection ${ }^{22}$. Specifically, fewer than $10^{7}$ cells were resuspended in $100 \mu$ l supplied buffer and incubated with $10 \mu \mathrm{l}$ CD45 MicroBeads (Miltenyi Biotec, Cologne, Germany) for $15 \mathrm{~min}$ at $4-8{ }^{\circ} \mathrm{C}$. After washing, the cell-MicroBead complexes were subjected to magnetic separation and the flow-through was resuspended in $\alpha$-MEM with $10 \%$ FBS and plated in culture dishes. To induce osteoblast differentiation, after reaching $100 \%$ confluency, cells were cultured in a-MEM supplemented with $10 \%$ FBS, $10 \mathrm{nM}$ dexamethasone, $50 \mu \mathrm{g} / \mathrm{ml}$ ascorbic acid and $10 \mathrm{mM} \beta$-glycerophosphate. Alkaline phosphatase (ALP) and Alizarin red staining were performed using 1-Step ${ }^{\text {TN }}$ NBT/ BCIP Substrate Solution (Thermo Fisher, Catalog number: 34042 ) and 0.5\% Alizarin red Staining solution ${ }^{11}$. Von Kossa staining was performed by placing cell culture plates submerged with $1 \%$ silver nitrate under the UV lamp for $10 \mathrm{~min}$.

In vitro assays. For transfection of miRNA or siRNA oligos, Lipofectamine RNAi MAX transfection reagent (Life Technologies, Grand Island, NY, USA) was used and siRNAs against Runx2, or Ngf (Sigma-Aldrich, St. Louis, MO, USA) or miR204 mimic (Dharmacon, Lafayette, CO, USA) were transfected at a concentration of $50 \mathrm{nM}$. Retroviral vectors for mouse $\mathrm{Ng}$ overexpression were constructed using the forward primer for $N g f-A$ (5'-TATAGAATTCATGCTGTGCCTCAAGCCA GTG-3') or for Ngf-B (5'-TATAGAATTCATGTCCATGTTGTTCTACACTC-3') and the reverse primer (5'-TATACTCGAGTCAGCCTCTTCTTGTAGCCTT- ${ }^{\prime}$, for both $\mathrm{Ng}$ f isoforms) to amplify two $\mathrm{Ng}$ isoforms which were digested with EcoRI and $\mathrm{XhoI}$ and then ligated to pMX vector. The preparation and infection of retrovirus were performed as follows ${ }^{53}$. Briefly, Plat-E packaging cells were cultured in DMEM (Life Technologies, Grand Island, NY, USA) with 10\% FBS and transfected with pMX retroviral vectors as described ${ }^{11}$. After $48 \mathrm{~h}$, the retroviral supernatant was collected and used to infect cells. Selection was performed using $5 \mu \mathrm{g} / \mathrm{ml}$ puromycin (EMD Millipore, Billerica, MA, USA) for 2-3 weeks. Western blot analysis was performed ${ }^{11}$, using antibodies against Runx2 (1:1,000, D130-3, MBL, Woburn, MA, USA), $\beta$-actin (1: 10,000, A5441, Sigma-Aldrich, St. Louis, MO, USA), phospho-Akt Thr308 (1: 1000, \#9275), total Akt (1: 3000, \#9272), phosphoPI3 Kinase p85 (Tyr458)/p55 (Tyr199) (1:1000, \#4228), and NGF (1:1000, \#2046) (the last four antibodies all from Cell Signaling, Danvers, MA, USA). Uncropped and unprocessed scans of the most important blots are supplied in the Source Data file. CLIP assays were performed as follows ${ }^{54}$. Briefly, CD45- ${ }^{-}$BMSCs were rinsed with PBS, then irradiated at $400 \mathrm{~mJ} \mathrm{~cm}^{-2}$ (setting of 4000 and $\lambda=254 \mathrm{~nm}$ ). Then we lysate the cells on ice for $10 \mathrm{~min}$ in $1 \mathrm{ml}$ buffer with $50 \mathrm{mM}$ Tris- $\mathrm{HCl}$ (pH 7.8), $300 \mathrm{mM} \mathrm{NaCl}, 1 \% \mathrm{NP}-40,5 \mathrm{mM}$ EDTA ( $\mathrm{pH} 8.0$ ) and $10 \%$ glycerol, $5 \mathrm{mM} \beta$ mercaptoethanol and protease inhibitors. Cleared lysates will be incubated with anti-Ago (mouse anti-Ago 2A8, Millipore, cat. no. MABE56) magnetic beads for 1 $\mathrm{h}$ and then washed with lysis buffer for three times. The Ago-binding beads were then treated with diluted RNase, T4 PNK, T4 RNA ligase and Shrimp Alkaline Phosphatase sequentially. Qiagen miRNeasy kit was used to purify RNA from the beads and reverse transcription was performed using qScript microRNA cDNA
Synthesis Kit (Quantabio). PCR were then performed using primer pairs covering miR-204 and putative binding sites of Runx2 3'-UTR. For profiling gene expressions, quantitative RT-PCR was performed, using the primer pairs for Runx2 (5' CAAGAAGGCTCTGGCGTTTA- $3^{\prime}$ and $5^{\prime}$-TGCAGCCTTAAATGACTCGG- $3^{\prime}$ ), Alp (5'-CCAACTCTTTTGTGCCAGAGA- $3^{\prime}$ and $5^{\prime}$-GGCTACATTGGTGTTGA GCTTTT-3'), osterix (5'-AGAGGTTCACTCGCTCTGACGA-3' and 5'-TTGC TCAAGTGGTCGCTTCTG-3'), osteocalcin (5'-CTGACCTCACAGATGCCAA GC-3' and 5'-TGGTCTGATAGCTCGTCACA AG-3'), p16 (5'-GAACTCTTTC GGTCGTACCC- $3^{\prime}$ and $5^{\prime}$-GTTCGAATCTGCACCGTAGT-3'), p21 (5'-CCTGG TGATGTCCGACCTG-3' and 5' ${ }^{\prime}$-CCATGAGCGCATCGCAATC- $\left.{ }^{\prime}\right), p 27\left(5^{\prime}\right.$-TC AAACGTGAGAGTGTCTAACG-3' and $5^{\prime}$-CCGGGCCGAAGAGATTTCTG-3'), p57 (5'-CGAGGAGCAGGACGAGAATC- ${ }^{\prime}$ ' and $5^{\prime}$-GAAGAAGTCGTTCGCA TTGGC-3'), Mmp13 (5'-CTTCTTCTTGTTGAGCTGGACTC-3' and 5'-CTGT GGAGGTCACTGTA GACT-3'), Adamts5 (5'-GGAGCGAGGCCATTTAC AAC- $3^{\prime}$ and $5^{\prime}$-CGTAGACAAGGTAG CCCACTTT- $\left.3^{\prime}\right), N g f\left(5^{\prime}\right.$-ACTGGAC TAAACTTCAGCAT TCC-3' and 5'-GGGCAGCTATT GGTGCAGTA-3') and $\beta$-actin (5'-GGCTGTATTCCCCTCCATCG- $3^{\prime}$ and $5^{\prime}$-CCAGTTGG TAACAATG CCATGT-3'). Specifically, for mouse $m i R-204$ and $m i R-211$ qRT-PCR, the reverse transcription primer is $m m u$ - $m i R-204 /-211-R T$ ( $5^{\prime}$-GTCGTATCCAGTGCAGGG TCCGAGG TATTCGCACTGGATACGACAGGCAW-3'), and the PCR primers are $m m u$ - $m i R-204$ forward (5'-GGGCTTCCCTTTGTCATCCTAT-3'), $m m u-m i R$ 211 forward (5'- GGGCTTCCCTTT GTCATCCTT-3') and the reverse primer (5'-CCAGTGCAGGGTCCGAGGT-3')

Micro-CT, histology, ISH, IHC and histomorphometry. We used a Scanco

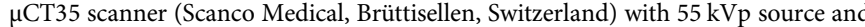
$145 \mu \mathrm{Amp}$ current for formalin-fixed mouse legs with a resolution of $10 \mu \mathrm{m}$. The scanned images from each group were evaluated at the same thresholds to allow three-dimensional structural rendering of each sample. For histology, ISH and IHC, tissues were fixed in $10 \%$ formalin, decalcified, and embedded in paraffin Serial sagittal sections of knee joints were cut every $3 \mu \mathrm{m}$ from the medial compartments. The sections were stained with Alcian blue/hematoxylin \& orange $\mathrm{G}$ (AB/H\&OG) for histological analysis. OARSI scoring was performed to evaluate knee joint AC destruction essentially ${ }^{55}$. Specifically, both medial femoral condyle and medial tibial plateau were analyzed through three-level sections of the joints and the severity of $\mathrm{OA}$ is expressed as the summed scores for the entire joint.

We performed in situ hybridizations on 3- $\mu \mathrm{m}$-thick sections using the microRNA ISH Buffer and Controls Kit (Exiqon, Vedbaek, Denmark). Briefly, sections were deparaffinized, permeabilized with Proteinase $\mathrm{K}$ for $10 \mathrm{~min}$ at $37^{\circ} \mathrm{C}$ treated with $3 \% \mathrm{H}_{2} \mathrm{O}_{2}$ to block endogenous peroxidase activity and then dehydrated before applying $40 \mathrm{nM}$ double-DIG LNA ${ }^{\mathrm{ma}}$ microRNA probe for $\mathrm{miR}$ 204 or $m i R-211$, both of which were purchased from Exiqon. Hybridization was performed for $1 \mathrm{~h}$ at $53^{\circ} \mathrm{C}$ for $m m u$-miR-204, and $48^{\circ} \mathrm{C}$ for $m m u-m i R-211$ respectively. After serial washes with $5 \times$ SSC buffer, $1 \times$ SSC buffer and $0.2 \times$ SSC buffer, sections were incubated with blocking solution for $15 \mathrm{~min}$, and then applied with anti-DIG-POD antibodies (Roche Applied Science, Indianapolis, IN, USA). Lastly, TSA-plus FITC substrate (Perkin Elmer, Waltham, MA, USA) was applied for detection of fluorescent signal and VECTASHIELD Mounting Medium with DAPI (Vector Laboratories, Burlingame, CA, USA) was used to mount the slides. For IHC, $3 \mu \mathrm{m}$ paraffin sections were heated at $95^{\circ} \mathrm{C}$ in Antigen Unmasking Solution (Vector Laboratories, Burlingame, CA, USA) for 10-15 min, and then sequentially treated with $3 \% \mathrm{H}_{2} \mathrm{O}_{2}, 0.5 \%$ Triton X-100, Avidin/Biotin Blocking Kit (Invitrogen, Carlsbad, CA, USA). After blocking with $10 \%$ normal goat serum (Vector Laboratories, Burlingame, CA, USA) for $1 \mathrm{~h}$, sections were treated with $1 /$ 200 anti-Runx2 antibody (D130-3, MBL, Woburn, MA, USA), 1/200 phospho-Akt antibody (\#9275, Cell Signaling, Danvers, MA, USA), 1/2000 PCNA antibody (ab29, Abcam, Cambridge, MA, USA), 1/200 MMP13 antibody (MAB13424, EMD Millipore, Billerica, MA, USA), 1/500 Adamts5 antibody (ab41037, Abcam, Cambridge, MA, USA), 1/1000 Cre antibody (69050, Novagen, Billerica, MA, USA), 1/200 NGF antibody (ab6199, Abcam, Cambridge, MA, USA), or 1/500 Adamts4 (ab185722, Abcam, Cambridge, MA, USA) antibody overnight at $4{ }^{\circ} \mathrm{C}$ and incubated with 1/400 secondary biotinylated goat anti-rabbit or anti-mouse antibody (Vector Laboratories, Burlingame, CA, USA) for $30 \mathrm{~min}$, followed by treatment with VECTASTAIN Elite ABC Kit (Vector Laboratories, Burlingame, CA, USA). IHC signals were revealed by ImmPACT DAB Peroxidase Substrate (Vector Laboratories, Burlingame, CA, USA). For fluorescence immunostaining, sections were incubated with 1/250 Ki67 antibody (ab15580, Abcam, Cambridge, MA, USA) overnight at $4{ }^{\circ} \mathrm{C}$ and then incubated with secondary antibody conjugated to Alexa Fluor 488 (Invitrogen, Carlsbad, CA, USA) for $30 \mathrm{~min}$. Images of histology, ISH and IHC were captured using CellSens Imaging Software (Olympus) on an Olympus BX43 microscope, or a Zeiss LSM700 confocal microscope. Histomorphometric measurements were performed using OsteoMeasure software (OsteoMetrics, Inc., Atlanta, GA, USA).

Flow cytometry. After 2-week culture in a-MEM plus 10\% FBS, mouse BMSCs were trypsinized, pelleted and then resuspended in PBS containing 2\% FBS to a concentration of $2 \times 10^{6}$ cells $/ \mathrm{ml}$. Freshly isolated bone marrow cells were resuspended to a concentration no more than $1 \times 10^{7}$ cells $/ \mathrm{ml}$. Cells were stained with anti-mouse CD45 PerCP-Cy5.5 (eBioscience, 45-0451-80), anti-mouse/rat CD29 PE-Cy7 (eBioscience, 25-0291-80), anti-mouse CD105 (Endoglin) PE (eBioscience, 
12-1051-81), and anti-mouse Ly-6A/E (Sca-1) APC (eBioscience, 17-5981-81), and analyzed with an LSRII cytometer (Becton Dickinson, San Jose, CA, USA). For BrdU Incorporation, the mice were intraperitoneally labeled twice (a $16-\mathrm{h}$ interval) with $1 \mathrm{mg} /$ mouse/injection BrdU and euthanized $2 \mathrm{~h}$ after the second injection. Freshly harvested bone marrow was treated with $1 \times$ RBC Lysis Buffer (eBiosciences, San Diego, CA, USA). The remaining cells were stained with anti-mouse CD45 PerCP-Cy5.5, anti-mouse/rat CD29 PE-Cy7, anti-mouse CD105 (Endoglin) $\mathrm{PE}$, and anti-mouse Ly-6A/E (Sca-1) Alexa Fluor 700 (eBioscience, 56-5981-82) before permeabilization, DNase treatment and staining with anti-BrdU APC according to the instruction of APC BrdU Flow Kits (BD Pharmagen, Franklin Lakes, NJ, USA). The percentage of BrdU-positive fractions was assessed using FlowJo analysis software (Tree Star, Ashland, OR, USA).

Animal behavioral tests. Testing for mechanical allodynia (von Frey sensitivity) was performed using a calibrated set of von Frey filaments (Stoelting, Wood Dale, $\mathrm{IL})^{56}$. Before the von Frey test, we allowed animals to adapt to the environment, including an elevated mesh platform for $15 \mathrm{~min}$. The set of von Frey filaments was used to poke from below to the hind paw to calculate the $50 \%$ force withdrawal threshold using an iterative approach. The tests were performed in a blind manner that the investigator is not aware of the identification of animals as well as the study groups.

Experimental design and statistical analyses. The sample size for each experiment was determined based on our previous experiences. All the data were expressed as mean \pm s.d., as indicated in the figure legends. Statistical analyses were completed with Prism GraphPad. Unpaired Student's $t$ test (for two groups), oneway ANOVA (for multiple groups) and two-way ANOVA (for multiple groups and time points) were used followed by the Tukey-Kramer test. $P<0.05$ was considered statistically significant.

\section{Data availability}

The data that support the findings of this study are available within the article and its Supplementary Information files or from the corresponding author upon reasonable request.

Received: 21 February 2019 Accepted: 24 May 2019

Published online: 28 June 2019

\section{References}

1. Hunter, D. J., Schofield, D. \& Callander, E. The individual and socioeconomic impact of osteoarthritis. Nat. Rev. Rheuma. 10, 437-441 (2014).

2. Lawrence, R. C. et al. Estimates of the prevalence of arthritis and other rheumatic conditions in the United States. Part II. Arthritis Rheum. 58, 26-35 (2008).

3. Goldring, S. R. \& Goldring, M. B. Changes in the osteochondral unit during osteoarthritis: structure, function and cartilage-bone crosstalk. Nat. Rev. Rheuma. 12, 632-644 (2016).

4. Loeser, R. F., Goldring, S. R., Scanzello, C. R. \& Goldring, M. B. Osteoarthritis: a disease of the joint as an organ. Arthritis Rheum. 64, 1697-1707 (2012).

5. Chevalier, X., Eymard, F. \& Richette, P. Biologic agents in osteoarthritis: hopes and disappointments. Nat. Rev. Rheuma. 9, 400-410 (2013).

6. Huang, J., Zhao, L. \& Chen, D. Growth factor signalling in osteoarthritis. Growth Factors 36, 187-195 (2018).

7. Jiang, Y. \& Tuan, R. S. Origin and function of cartilage stem/progenitor cells in osteoarthritis. Nat. Rev. Rheuma. 11, 206-212 (2015).

8. van der Kraan, P. M. \& van den Berg, W. B. Osteophytes: relevance and biology. Osteoarthr. Cartil. 15, 237-244 (2007).

9. Asahara, H. Current status and strategy of microRNA research for cartilage development and osteoarthritis pathogenesis. J. Bone Metab. 23, 121-127 (2016).

10. Swingler, T. E. et al. The expression and function of microRNAs in chondrogenesis and osteoarthritis. Arthritis Rheum. 64, 1909-1919 (2012).

11. Huang, J., Zhao, L., Xing, L. \& Chen, D. MicroRNA-204 regulates Runx2 protein expression and mesenchymal progenitor cell differentiation. Stem Cells 28, 357-364 (2010).

12. Xu, G., Chen, J., Jing, G. \& Shalev, A. Thioredoxin-interacting protein regulates insulin transcription through microRNA-204. Nat. Med. 19, 1141-1146 (2013).

13. Vikram, A. et al. Vascular microRNA-204 is remotely governed by the microbiome and impairs endothelium-dependent vasorelaxation by downregulating Sirtuin1. Nat. Commun. 7, 12565 (2016).

14. Conte, I. et al. MiR-204 is responsible for inherited retinal dystrophy associated with ocular coloboma. Proc. Natl. Acad. Sci. USA 112, E3236-E3245 (2015).
15. Courboulin, A. et al. Role for miR-204 in human pulmonary arterial hypertension. J. Exp. Med. 208, 535-548 (2011).

16. Levy, C. et al. Intronic miR-211 assumes the tumor suppressive function of its host gene in melanoma. Mol. Cell 40, 841-849 (2010).

17. Schwenk, F., Baron, U. \& Rajewsky, K. A cre-transgenic mouse strain for the ubiquitous deletion of loxP-flanked gene segments including deletion in germ cells. Nucleic Acids Res. 23, 5080-5081 (1995).

18. Sampson, E. R. et al. Establishment of an index with increased sensitivity for assessing murine arthritis. J. Orthop. Res. 29, 1145-1151 (2011).

19. Logan, M. et al. Expression of Cre Recombinase in the developing mouse limb bud driven by a Prxl enhancer. Genesis 33, 77-80 (2002).

20. Yue, R., Zhou, B. O., Shimada, I. S., Zhao, Z. \& Morrison, S. J. Leptin receptor promotes adipogenesis and reduces osteogenesis by regulating mesenchymal stromal cells in adult bone marrow. Cell Stem Cell 18, 782-796 (2016).

21. Pritzker, K. P. et al. Osteoarthritis cartilage histopathology: grading and staging. Osteoarthr. Cartil. 14, 13-29 (2006).

22. Zhao, L. et al. Tumor necrosis factor inhibits mesenchymal stem cell differentiation into osteoblasts via the ubiquitin E3 ligase Wwp1. Stem Cells 29, 1601-1610 (2011).

23. Chi, S. W., Zang, J. B., Mele, A. \& Darnell, R. B. Argonaute HITS-CLIP decodes microRNA-mRNA interaction maps. Nature 460, 479-486 (2009).

24. Czech, B. \& Hannon, G. J. Small RNA sorting: matchmaking for Argonautes. Nat. Rev. Genet. 12, 19-31 (2011).

25. Lin, A. C. et al. Modulating hedgehog signaling can attenuate the severity of osteoarthritis. Nat. Med. 15, 1421-1425 (2009).

26. Wang, X. et al. Regulation of MMP-13 expression by RUNX2 and FGF2 in osteoarthritic cartilage. Osteoarthr. Cartil. 12, 963-973 (2004).

27. Kamekura, S. et al. Contribution of runt-related transcription factor 2 to the pathogenesis of osteoarthritis in mice after induction of knee joint instability. Arthritis Rheum. 54, 2462-2470 (2006).

28. Liao, L. et al. Deletion of Runx2 in articular chondrocytes decelerates the progression of DMM-induced osteoarthritis in adult mice. Sci. Rep. 7, 2371 (2017).

29. Sakaguchi, Y., Sekiya, I., Yagishita, K. \& Muneta, T. Comparison of human stem cells derived from various mesenchymal tissues: superiority of synovium as a cell source. Arthritis Rheum. 52, 2521-2529 (2005).

30. Manning, B. D. \& Cantley, L. C. AKT/PKB signaling: navigating downstream. Cell 129, 1261-1274 (2007).

31. Engelman, J. A., Luo, J. \& Cantley, L. C. The evolution of phosphatidylinositol 3-kinases as regulators of growth and metabolism. Nat. Rev. Genet. 7, 606-619 (2006).

32. Hasuwa, H., Ueda, J., Ikawa, M. \& Okabe, M. miR-200b and miR-429 function in mouse ovulation and are essential for female fertility. Science 341, 71-73 (2013).

33. Zhao, L. et al. Exploration of CRISPR/Cas9-based gene editing as therapy for osteoarthritis. Ann. Rheum. Dis. 78, 676-682 (2019).

34. Khoury, M. et al. Adeno-associated virus type 5-mediated intraarticular administration of tumor necrosis factor small interfering RNA improves collagen-induced arthritis. Arthritis Rheum. 62, 765-770 (2010).

35. Miyaki, S. et al. MicroRNA-140 plays dual roles in both cartilage development and homeostasis. Genes Dev. 24, 1173-1185 (2010).

36. Yang, S. et al. Hypoxia-inducible factor-2alpha is a catabolic regulator of osteoarthritic cartilage destruction. Nat. Med. 16, 687-693 (2010).

37. Saito, T. et al. Transcriptional regulation of endochondral ossification by HIF2alpha during skeletal growth and osteoarthritis development. Nat. Med. 16, 678-686 (2010)

38. Zhang, M. et al. Induced superficial chondrocyte death reduces catabolic cartilage damage in murine posttraumatic osteoarthritis. J. Clin. Invest. 126, 2893-2902 (2016).

39. Shen, J. et al. Deletion of the transforming growth factor beta receptor type II gene in articular chondrocytes leads to a progressive osteoarthritis-like phenotype in mice. Arthritis Rheum. 65, 3107-3119 (2013).

40. Rockel, J. S. et al. Hedgehog inhibits beta-catenin activity in synovial joint development and osteoarthritis. J. Clin. Invest. 126, 1649-1663 (2016).

41. Yang, X. et al. TGF-beta/Smad3 signals repress chondrocyte hypertrophic differentiation and are required for maintaining articular cartilage. J. Cell Biol. 153, 35-46 (2001).

42. De Bari, C., Dell'Accio, F., Tylzanowski, P. \& Luyten, F. P. Multipotent mesenchymal stem cells from adult human synovial membrane. Arthritis Rheum. 44, 1928-1942 (2001).

43. Segawa, Y. et al. Mesenchymal stem cells derived from synovium, meniscus, anterior cruciate ligament, and articular chondrocytes share similar gene expression profiles. J. Orthop. Res. 27, 435-441 (2009).

44. Alsalameh, S., Amin, R., Gemba, T. \& Lotz, M. Identification of mesenchymal progenitor cells in normal and osteoarthritic human articular cartilage. Arthritis Rheum. 50, 1522-1532 (2004).

45. Bernardo, M. E. \& Fibbe, W. E. Mesenchymal stromal cells: sensors and switchers of inflammation. Cell Stem Cell 13, 392-402 (2013). 
46. Mendicino, M., Bailey, A. M., Wonnacott, K., Puri, R. K. \& Bauer, S. R. MSCbased product characterization for clinical trials: an FDA perspective. Cell Stem Cell 14, 141-145 (2014).

47. Trounson, A. \& McDonald, C. Stem cell therapies in clinical trials: progress and challenges. Cell Stem Cell 17, 11-22 (2015).

48. Liu, P., Jenkins, N. A. \& Copeland, N. G. A highly efficient recombineeringbased method for generating conditional knockout mutations. Genome Res. 13, 476-484 (2003).

49. Chan, W. et al. A recombineering based approach for high-throughput conditional knockout targeting vector construction. Nucleic Acids Res. 35, e64 (2007).

50. Farley, F. W., Soriano, P., Steffen, L. S. \& Dymecki, S. M. Widespread recombinase expression using FLPeR (flipper) mice. Genesis 28, 106-110 (2000).

51. Futami, I. et al. Isolation and characterization of multipotential mesenchymal cells from the mouse synovium. PLoS ONE 7, e45517 (2012).

52. Gosset, M., Berenbaum, F., Thirion, S. \& Jacques, C. Primary culture and phenotyping of murine chondrocytes. Nat. Protoc. 3, 1253-1260 (2008).

53. Zhao, L. et al. Smurf1 inhibits mesenchymal stem cell proliferation and differentiation into osteoblasts through JunB degradation. J. Bone Min. Res. 25, 1246-1256 (2010).

54. Helwak, A. \& Tollervey, D. Mapping the miRNA interactome by cross-linking ligation and sequencing of hybrids (CLASH). Nat. Protoc. 9, 711-728 (2014).

55. Glasson, S. S., Chambers, M. G., Van Den Berg, W. B. \& Little, C. B. The OARSI histopathology initiative-recommendations for histological assessments of osteoarthritis in the mouse. Osteoarthr. Cartil. 18(Suppl 3), S17-S23 (2010).

56. Chaplan, S. R., Bach, F. W., Pogrel, J. W., Chung, J. M. \& Yaksh, T. L. Quantitative assessment of tactile allodynia in the rat paw. J. Neurosci. Methods 53, 55-63 (1994).

\section{Acknowledgements}

We would like to express our gratitude to Ms. Lily Yu, for her assistance on processing and staining histological samples, and Mr. Jeffrey Martinson, for his help on flow cytometry. This work was supported by the National Institutes of Health Grants R01AR054465 and R01AR070222 to D.C. and the Rush 2015 Schweppe/Armour bequest Pilot Project to L.Z. G.X. was partially supported by NSFC grant 81630066 .

\section{Author contributions}

J.H. generated $m i R-204$ and $m i R-211$ floxed mice, designed the research and performed experiments, analyzed data and wrote the manuscript. L.Z. designed and performed experiments, analyzed data and revised the manuscript. Y.F. and L.L. performed mouse surgery and intraarticular injection. G.X. and P.X.M. provided expertise and feedback. D.C. designed the project, analyzed the results and revised the manuscript.

\section{Additional information}

Supplementary Information accompanies this paper at https://doi.org/10.1038/s41467019-10753-5.

Competing interests: The authors declare no competing interests.

Reprints and permission information is available online at http://npg.nature.com/ reprintsandpermissions/

Peer review information: Nature Communications thanks John Rossi, Hiroshi Asahara and other anonymous reviewer(s) for their contribution to the peer review of this work.

Publisher's note: Springer Nature remains neutral with regard to jurisdictional claims in published maps and institutional affiliations.

(c) (i) Open Access This article is licensed under a Creative Commons Attribution 4.0 International License, which permits use, sharing, adaptation, distribution and reproduction in any medium or format, as long as you give appropriate credit to the original author(s) and the source, provide a link to the Creative Commons license, and indicate if changes were made. The images or other third party material in this article are included in the article's Creative Commons license, unless indicated otherwise in a credit line to the material. If material is not included in the article's Creative Commons license and your intended use is not permitted by statutory regulation or exceeds the permitted use, you will need to obtain permission directly from the copyright holder. To view a copy of this license, visit http://creativecommons.org/ licenses/by/4.0/.

(C) The Author(s) 2019 